\title{
Hydrodynamic and Polyelectrolyte Properties of Actin Filaments: Theory and Experiments
}

\author{
Ernesto Alva, Annitta George, Lorenzo Brancaleon, and Marcelo Marucho* \\ January 12, 2022
}

Department of Physics and Astronomy, University of Texas at San Antonio, TX 78249, USA

\begin{abstract}
Actin filament's polyelectrolyte and hydrodynamic properties, their interactions with the biological environment, and external force fields play an essential role in their biological activities in eukaryotic cellular processes. In this article, we introduce a unique approach that combines dynamics and electrophoresis light scattering experiments, an extended semiflexible worm-like chain model, and an asymmetric polymer length distribution theory to characterize the polyelectrolyte and hydrodynamic properties of actin filaments in aqueous electrolyte solutions. We used the same sample and experimental conditions and considered several g-actin and polymerization buffers to elucidate the impact of their chemical composition, reducing agents, $\mathrm{pH}$ values, and ionic strengths on the filament translational diffusion coefficient, electrophoretic mobility, structure factor, asymmetric length distribution, effective filament diameter, electric charge, zeta potential, and semiflexibility. Compared to those values obtained from molecular structure models, our results revealed a lower value of the effective G-actin charge and a more significant value of the effective filament diameter due to the formation of the double layer of the electrolyte surrounding the filaments. Additionally, compared to the values usually reported from electron micrographs, the lower values of our results for the persistence length and average contour filament length agrees with the significant difference in the association rates at the filament ends that shift to sub-micro lengths, the maximum of the length distribution.
\end{abstract}

\section{Introduction}

Actin filaments (F-actins) are highly-charged double-stranded rod-like polyelectrolytes formed by the polymerization of G-actin proteins. Cytoskeleton filaments are essential for various biological activities in eukaryotic cellular processes. These filaments are usually

\footnotetext{
*Corresponding author Email: marcelo.marucho@utsa.edu
} 
organized into higher-order structures, forming bundles and networks which provide mechanical support, determine cell shape, and allow movement of the cell surface, thereby enabling cells to migrate, engulf particles, and divide. One major challenge in biophysics is to elucidate the role of the polyelectrolyte properties of the filaments, their interactions with the biological environment, and external force fields on their higher-order structure formation and stability. Indeed, it is imperative and crucial for understanding emergent or macroscopic properties of these systems. During the last few decades, a substantial amount of research has been done on the diffusion coefficient, shear modulus, second virial coefficient, and electrophoretic mobility of actin filaments[1]-[10]. Nevertheless, the underlying biophysical principles and molecular mechanisms that support the polyelectrolyte nature of $\mathrm{F}$-actins and their properties still remain elusive. This uncertainty is due to the lack of unicity, consistency, and sometimes accuracy in the methodologies, techniques, and sample preparation protocols used decades ago in scattering experiments to produce meaningful, reproducible results. At the same time, the optimization of actin filament and electrolyte models and sophisticated molecular-level kinetic theories used to characterize these macroscopic properties became burdensome due to the limited access and availability of high-performance numerical algorithms and powerful computational resources.

Nowadays, modern Dynamic Light Scattering (DLS) and Electrophoresis Light Scattering (ELS) instruments are robust and accurate tools to characterize hydrodynamics properties of polydisperse charged biomolecules even at low concentrations and using small sample volume. These non-invasive, susceptible, and resolution instruments use advanced technology and multi-functional software to measure the translational diffusion coefficient, second virial coefficient, and electrophoresis mobility with high accuracy and reproducibility. Additionally, the state-of-the-art scientific software and multi-core cluster computers provide reliable tools and computing efficiency required to characterize these complex systems. DLS and ELS experiments also allow for accurate measurement of model parameters if the number of these parameters is small and the approach is adequate to characterize the hydrodynamic and polyelectrolyte properties of the biomolecules in solutions[11], [12].

In this article, we introduce a unique approach that combines light scattering experiments and optimized theoretical approaches to characterize actin filaments' polyelectrolyte and hydrodynamic properties. We used Malvern ULTRA Zetasizer instrument to measure actin filament's translational diffusion coefficient and electrophoretic mobility at low protein concentration. We developed a novel sample preparation protocol based on bio-statistical tools[13] to minimize errors and assure reproducibility in our results. This protocol was used for all the experiments. We considered three different buffers, g-actin and polymerization, used in previous works[8]-[10] to elucidate the impact of their chemical composition, reducing agents, $\mathrm{pH}$ values, and ionic strengths on the filament properties. We also performed protein dialysis[14] and spectrophotometric[15] techniques to measure the protein concentration in our samples.

Additionally, we used a novel multi-scale approach to calculate the translational diffusion coefficient and electrophoretic mobility of polydisperse actin filaments in aqueous salt solutions. The monodisperse translational diffusion coefficient calculations are based on the Stokes-Einstein formulation[16] and a modified wormlike chain (WLC) model for the 


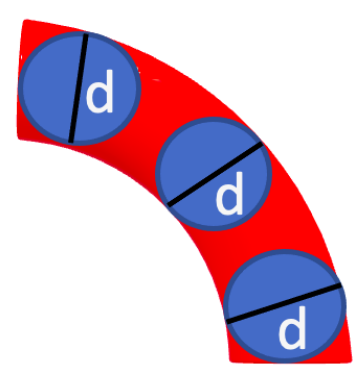

Figure 1: Cylindrical model of the wormlike chain enclosing a number of beads representing the actin filaments where the persistence length is a predominant factor in the theory of diffusion coefficient as well as the electrophoretic mobility.

hydrodynamic radius[17]. The monodisperse electrophoretic mobility calculations are carried out using a linear polymer representation of the WLC, which accounts for the balance between forces acting on each chain's monomer. This model and the Routine-Prager tensor for hydrodynamic interactions calculation are used to take the orientational average over all possible polymer conformations in the low electric field approximation[18]. An asymmetric, exponential length distribution is used to characterize the actin filament polydispersity and the different increasing rate lengths of barbed and pointed ends[19]. We used the length distribution to take the filament length average over the monodisperse translational diffusion coefficient and electrophoretic mobility expressions. The resulting expressions for the polydisperse translational diffusion coefficient and electrophoretic mobility depend on the persistence length, the effective filament diameter, the monomer charge, and the increasing rate length of barbed and pointed ends of the filaments. We used Mathematica software, a fitting approach, and multi-core computers to find optimal values for these parameters that better reproduce the translational diffusion coefficient and electrophoretic mobility values obtained experimentally.

\section{THEORETICAL APPROACH}

\section{Diffusion Theory}

The Stokes-Einstein formulation provides the following expression to calculate the monodisperse translational diffusion coefficient of colloidal particles of any shape

$$
D\left(L, L_{p}, d\right)=\frac{k_{B} T}{6 \pi \eta R_{h}\left(L, L_{p}, d\right)}
$$

where $k_{B}, T$, and $\eta$ represent the Boltzmann constant, temperature, and viscosity of the dispersant, respectively. The hydrodynamic radius, $R_{h}$, is also a factor in eq. (1) which depends on the contour length, $L$, persistence length, $L_{p}$, and diameter of the filament, $d$.

The hydrodynamic radius is calculated using Mansfield's approach for transport properties of semiflexible polymers[17]. The approach is based on the orientational preaveraging approximation. The charge distribution over the surface of an arbitrary shaped 
charged conductor is proportional to the Stokes-flow force distribution over the surface of a rigid body of the same size and shape as the conductor. Additionally, a cylindrical model of the WLC is used to account for not only the persistence, $L_{p}$, and contour length, $L$, but also the diameter of the chain, $d=2 a$. (see Figure 1). As a result, the expression for the hydrodynamic radius of a semiflexible polymer is given by

$$
R_{h}\left(L, L_{p}, a\right)=\frac{1+(0.03801) r_{c}^{-0.9212}}{1+(0.07204) r_{c}^{-1.0204}} R_{h_{\text {rod }}}
$$

where

$$
R_{h_{\text {rod }}}=\left[\frac{1}{2}(1+\delta)\left[\ln u+\ln 4-1-\frac{3.95}{(\ln u)^{2}}+\frac{16.18}{(\ln u)^{3}}-\frac{16}{(\ln u)^{4}}\right]^{-1}\right] L
$$

is the corresponding expression for a rigid polymer, and

$$
r_{c}=\epsilon \delta^{-0.134}, \quad r_{\alpha}=\epsilon \exp \left[(3.106) \delta^{1.213}\right], \quad u=\frac{L / 2+a}{a}=\delta^{-1}+1, \quad \epsilon=\frac{L_{p}}{L}, \quad \delta=\frac{d}{L}
$$

The approach also provides an accurate expression for the electrical polarizability, $<\alpha>[17]$.

$$
\begin{gathered}
L^{3} \frac{\pi}{18}(1+\delta)^{3}\left[\ln (u)+\ln (4)-\frac{7}{4}\right]^{-1}, \\
L^{3} \frac{\pi}{18}(1+\delta)^{3}\left[\ln (u)+\ln (4)-\frac{7}{4}-\frac{4.53}{[\ln (u)]^{1.72}}+\frac{18.3}{[\ln (u)]^{2.72}}-\frac{18}{[\ln (u)]^{3.72}}\right], \quad 10^{-4} \leq \delta \leq 10^{-1} \\
<\alpha>\left(L, L_{p}, a\right)=\frac{<\alpha>_{\text {rod }}\left(1-0.005690 r_{\alpha}^{-0.8350}\right)}{1+0.2028 r_{\alpha}^{-1.0335}}
\end{gathered}
$$

and the radius of gyration, $R_{g}$

$$
\begin{aligned}
& R_{g, \text { thin }}^{2}=\begin{array}{cc}
L^{2}\left[\frac{\varepsilon}{3}-\varepsilon^{2}+2 \varepsilon^{3}-2 \varepsilon^{4}\left[1-\exp \left(-\frac{1}{\varepsilon}\right)\right]\right], & \varepsilon \leq 10 \\
L^{2} \sum_{k} \frac{2}{(k+4) !}\left(-\frac{1}{\varepsilon}\right)^{k}, & \varepsilon>10
\end{array} \\
& R_{g, r o d}^{2}=L^{2} \frac{1+2 \delta+3 \delta^{2}+\frac{6}{5} \delta^{3}}{12+8 \delta} \\
& R_{g}\left(L, L_{p}, a\right)=\frac{\sqrt{12} R_{g, \text { thin }} R_{g, \text { rod }}}{L}
\end{aligned}
$$

The approach generalizes previous results including Yamakawa-Fuji's theory[20] which is accurate for long chains only. This theory was successfully tested against experimental data on double stranded DNA. 


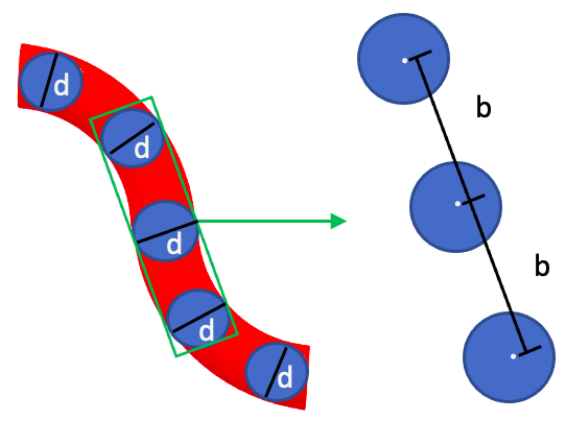

Figure 2: Conformation of a chain beads representing an actin filament where external forces and an electric field applied to beads form an arbitrary distribution of bending and structural conformations. The distance between beads is defined as the parameter $b$, as seen on the right side of the image. The chain of beads is under the effect of an electric field represented by 'E'.

\section{Electrophoretic Mobility Theory}

We used Völkel's theory[18] to calculate the monodisperse electrophoretic mobility of stiffcharged molecules in solution. In the low external electric field limit, the WLC model can be accurately represented by a semiflexible Gaussian chain consisting of $N$ monomers (beads) of radius $a$, charge $q$, center-to-center monomer separation distance $b=4 a$, and persistence length $L_{p}$. Whereas the aqueous electrolyte solution is considered as a homogeneous, incompressible solvent with viscosity, $\eta$, and arbitrary inverse Debye length, $\kappa$.

As a unique feature, this actin filament representation accounts for all the forces acting on each bead (see Figure 2) such as bending and stretching forces due to intramolecular potentials and the excluded volume and Coulomb interactions, and externally applied electric fields. Additionally, the Rotne-Prager tensor approximation[18] is used to calculate the hydrodynamic interactions between monomers, the counterion relaxation effects are neglected, and the Debye-Hückel potential[21] is used in the electrical force calculations. More, the Kirkwood-Riseman orientational pre-averaging approximation[22] is used to account for all the different rotational conformations of a filament. As a result, the expression for the monodisperse electrophoretic mobility expression for single actin filaments reads,

$$
\mu\left(N, q, a, L_{p}\right)=\frac{1}{N} \sum_{i=1, j=1}^{N}\left[<D\left(R_{i j}\right)>q-\frac{1}{6 \pi \eta}<R_{i j}^{-1}>_{b} q+\frac{\epsilon}{6 \pi \eta}<\psi\left(R_{i j}\right)>_{b}\right],
$$

where,

$$
\begin{aligned}
<D\left(R_{i j}\right)> & =\frac{1}{6 \pi \eta a}\left[\operatorname{erf}\left(\sqrt{u_{i j}}\right)+\frac{e^{-u_{i j}}-1}{\sqrt{\pi u_{i j}}}\right], \\
& <R_{i j}^{-1}>_{b}=\sqrt{\frac{u_{i j}}{\pi} e^{-u_{i j}}},
\end{aligned}
$$




$$
\begin{gathered}
<\psi\left(R_{i j}\right)>_{b}=\frac{q}{6 \pi \eta a \epsilon} \sqrt{\frac{u_{i j}}{\pi}} e^{-u_{i j}}\left\{e^{-\kappa b}\left[1-\frac{\kappa b}{\kappa b+2 u_{i j}} \operatorname{erfc}\left(k+\sqrt{u_{i j}}\right)\right]\right\}, \\
u_{i j}=\frac{\alpha b^{2}}{b|i-j|-\sqrt{\frac{\beta}{\alpha}}\left(1-e^{-b|i-j| \sqrt{\frac{\alpha}{\beta}}}\right)}
\end{gathered}
$$

In the previous expressions, $\left\langle\ldots>\right.$ represents the orientational average, $k=\kappa b / 2 \sqrt{u_{i j}}$, $\alpha=3 / 4 L_{p}, \beta=3 L_{p} / 4$, and $\operatorname{erf}(x)$ and $\operatorname{erfc}(x)$ are the error and complementary error functions, respectively. The first term in eq. (10) represents the monodisperse electrophoretic mobility's contribution to the hydrodynamic interactions between monomers. The second and third terms account for the electrostatic screen generated by the electrolyte on the monomer charges.

The formulation has been validated for the single- and double-stranded DNA and numerical simulations, and it generalizes previous approaches, including the method introduced by Muthukumar[23] and Oseen[24].

\section{Length Distribution Theory}

The G-actin polymerization in aqueous electrolyte solutions generates filaments of different contour lengths[9], [10], [25], [26] (see Figure 3). The filament length distribution represents the number of actin filaments with a given contour length $L$. It depends on $G$ actin concentration, polymerization buffer, ionic strength, and the significant, independent, asymmetric, length growth rate $\lambda_{+}$and $\lambda_{-}$from both barbed and pointed ends, respectively[5], [27]. The filaments polymerize bidirectionally with the rate at the fast end about ten times larger than at the slow end. The fast end is the barbed end; the slow end is the pointed end[27]. In this work, we used the generalized Schulz distribution $Y\left(L, \lambda_{+}, \lambda_{-}, b i\right)$ introduced by Jeune-Smith[19] for cytoskeleton filaments

$$
Y\left(L, \lambda_{+}, \lambda_{-}, b i\right)=(1-b i) * Y_{s}\left(L, \lambda_{+}, \lambda_{-}\right)+2 b i Y_{s}\left(2 L, \lambda_{+}, \lambda_{-}\right)
$$

where,

$$
Y_{s}\left(L, \lambda_{+}, \lambda_{-}\right)=\frac{\lambda_{+} \lambda_{-}}{\lambda_{-}-\lambda_{+}}\left(e^{-\lambda_{+} L}-e^{-\lambda_{-} L}\right)
$$

and the parameter bi represents the fraction of broken filaments which accounts for the shearing effects. Furthermore, we considered the experimental relationship between associate rates $\lambda_{+}=10 \lambda_{-}[5]$.

The generalized Schulz distribution was validated on microtubules polymerized in vitro, and it generalizes previous approaches, including the classic Schulz distribution theory developed for polymers with equal length distributions at each end [28]. In this work, we do not consider annealing effects on the actin filament length distribution since the sample preparation protocol used in the experimental work is designed to minimize breaking and the combination of actin filaments. 

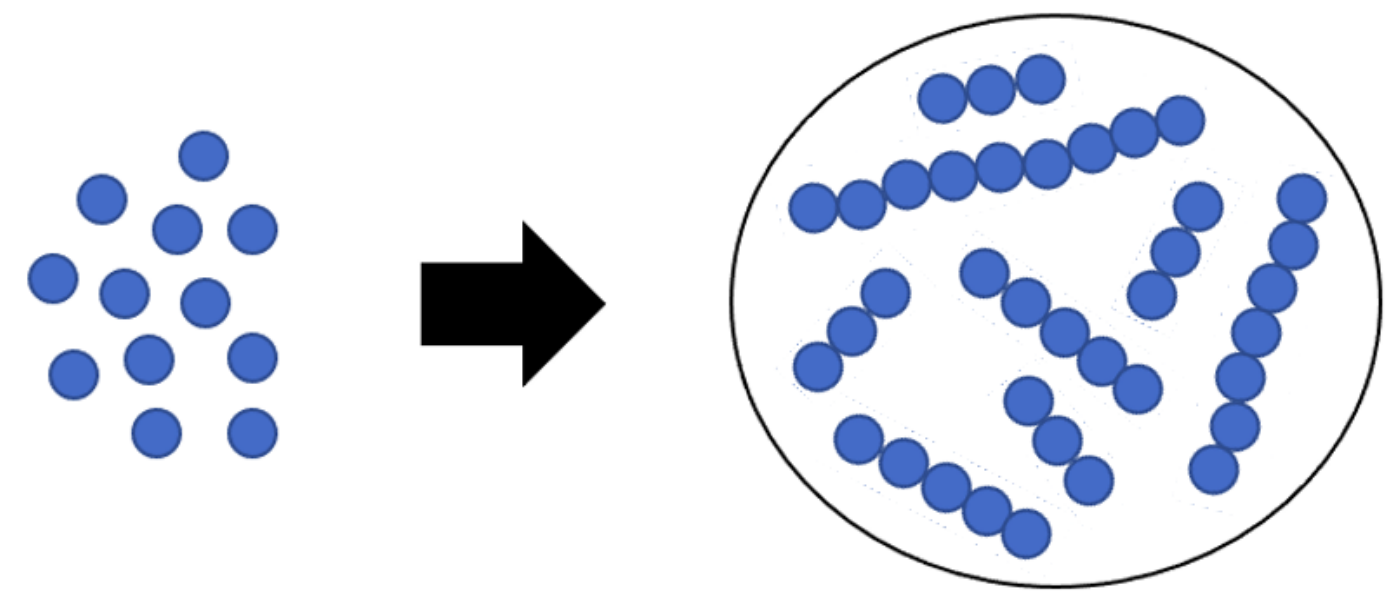

Figure 3: Schematic representation of G-actin monomers (left) that polymerizes into many different actin filament lengths (right). This account for the polydispersity that we encounter in a real system.

\section{Polydispersity Theory}

The relative contribution of individual biomolecules to some macroscopic properties, including those measured by light scattering experiments[9], is often proportional to their mass fractions $M$, in such a way that larger biomolecules gain greater significance. Considering the assumption where all actin filaments have the same diameter and density, the mass fraction of any actin filament becomes proportional to the squared contour length. Thus, we used the actin filaments weight function $M \sim L^{2}$ and the length distribution given by eq. (16) to take the length average of eqs. (1), (10), (6), and (9). The resulting expressions for the polydisperse translational diffusion coefficient, electrophoretic mobility, gyration radius, and polarizability for actin filaments in aqueous salt solutions read

$$
\begin{aligned}
\mu_{\text {avg }}\left(\lambda_{+}, \lambda_{-}, a, q, L_{p}, b i\right) & =\frac{\sum_{i=1}^{N} \mu\left(N_{i}, q, a, L_{p}\right)\left(\frac{N_{i}}{m d}\right)^{2} Y\left(\frac{N_{i}}{m d}, \lambda_{+}, \lambda_{-}, b i\right)}{\sum_{i=1}^{N}\left(\frac{N_{i}}{m d}\right)^{2} Y\left(\frac{N_{i}}{m d}, \lambda_{+}, \lambda_{-}, b i\right)} \\
D_{\text {avg }}\left(\lambda_{+}, \lambda_{-}, a, L_{p}, b i\right) & =\frac{\sum_{i=1}^{N} D\left(\frac{N_{i}}{m d}, L_{p}, a\right)\left(\frac{N_{i}}{m d}\right)^{2} Y\left(\frac{N_{i}}{m d}, \lambda_{+}, \lambda_{-}, b i\right)}{\sum_{i=1}^{N}\left(\frac{N_{i}}{m d}\right)^{2} Y\left(\frac{N_{i}}{m d}, \lambda_{+}, \lambda_{-}, b i\right)} \\
R_{g_{\text {avg }}}\left(\lambda_{+}, \lambda_{-}, a, L_{p}, b i\right) & =\frac{\sum_{i=1}^{N} R_{g}\left(\frac{N_{i}}{m d}, L_{p}, a\right)\left(\frac{N_{i}}{m d}\right)^{2} Y\left(\frac{N_{i}}{m d}, \lambda_{+}, \lambda_{-}, b i\right)}{\sum_{i=1}^{N}\left(\frac{N_{i}}{m d}\right)^{2} Y\left(\frac{N_{i}}{m d}, \lambda_{+}, \lambda_{-}, b i\right)} \\
<\alpha>_{\text {avg }}\left(\lambda_{+}, \lambda_{-}, a, L_{p}, b i\right) & =\frac{\sum_{i=1}^{N}<\alpha>\left(\frac{N_{i}}{m d}, L_{p}, a\right)\left(\frac{N_{i}}{m d}\right)^{2} Y\left(\frac{N_{i}}{m d}, \lambda_{+}, \lambda_{-}, b i\right)}{\sum_{i=1}^{N}\left(\frac{N_{i}}{m d}\right)^{2} Y\left(\frac{N_{i}}{m d}, \lambda_{+}, \lambda_{-}, b i\right)}
\end{aligned}
$$

In the previous equations, we used the relationship between the degree of polymerization (the number of G-actin monomers per micrometer) $m d$, the monomers number $N_{i}$, and the contour length $L_{i}=N_{i} / m d$. Additionally, we used the experimental value $m d=370 / \mu m$ [9], and generated a histogram for the filament contour length distribution 
a)

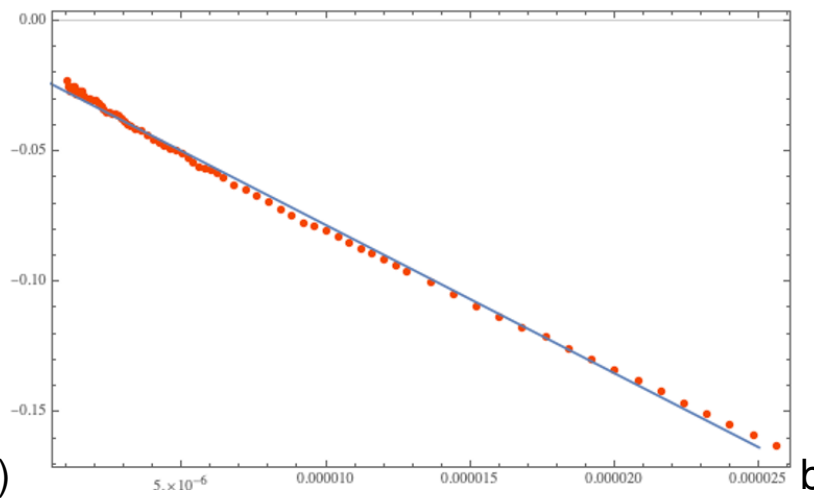

b)

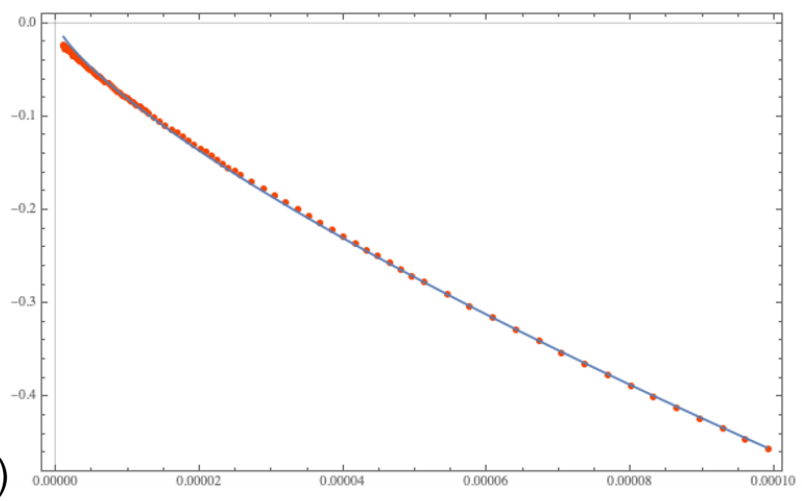

Figure 4: Mathematic Fitting Approach: a) Initial decay rate $\left(\gamma_{0}\right)$, and b) Persistence length $\left(L_{p}\right)$ fitting Mathematica plots using eqs (21) and (22).

using $0.2 \mu \mathrm{m}$ intervals (bins): $0.2 \mu \mathrm{m}, 0.4 \mu \mathrm{m}, 0.6 \mu \mathrm{m}, . ., 5.4 \mu \mathrm{m}$. Thus, the summation in eqs. (17) and (18) was performed over the monomers number $N_{i}=74(i-1)$, with $i=2,3, . ., 28$.

\section{Dynamic structure factor theory for semiflexible polymers}

We used Kroy's theory[11] to calculate the first cumulant (initial decay rate) $\gamma_{o}$ and the dynamic structure factor $g_{1}\left(k_{s}, t\right)$. The approach is based on the WLC model and the theory for Brownian particles in hydrodynamic solvent in dilute solutions. For short times, $g_{1}\left(k_{s}, t\right) \sim \exp \left[-\gamma_{o} t\right]$ with the initial decay rate given by,

$$
\gamma_{o}=-\left.\frac{d \ln \left[g_{1}\left(k_{s}, t\right)\right]}{d t}\right|_{t=0}=\frac{k_{B} T}{6 \pi^{2} \eta} k_{s}^{3}\left(\frac{5}{6}-\ln \left(k_{s} 2 a\right)\right)
$$

where $k_{s}=\frac{4 \pi}{\lambda} \sin (\theta / 2)$ is the scattering wave number, $\lambda$ the wavelength, $\theta$ the scattering angle, and $a$ the filament radius. Whereas the time decay of the dynamic structure factor is given by the stretched exponential approximation

$$
\frac{g_{1}\left(k_{s}, t\right)}{g_{1}\left(k_{s}, 0\right)} \simeq \exp \left[-\frac{\Gamma(1 / 4)}{3 \pi}\left[\frac{k_{B} T}{4 \pi \eta}\left(\frac{5}{6}-\ln \left(k_{s} 2 a\right)\right)\right]^{3 / 4} \frac{k_{s}^{2} t^{3 / 4}}{L_{p}^{1 / 4}}\right]
$$

Additionally, the initial decay rate $\gamma_{o}$ and the dynamic structure factor $g_{1}(k, t)$ can be obtained from the normalized autocorrelation function $g_{2}\left(k_{s}, t\right)$ measured in DLS experiments [12], [29].

$$
g_{2}\left(k_{s}, t\right)=1+\beta\left[g_{1}\left(k_{s}, t\right)\right]^{2}
$$

where $\beta$ is a constant depending on the optical system used and can be determined by using the property $g_{2}\left(k_{s}, t \rightarrow 0\right) \rightarrow 1$. 


\section{Zeta Potential}

We used Oshima's approach[30], and the values for the electrophoretic mobility were measured experimentally to estimate the filament zeta potential, $\zeta$. Considering actin filaments oriented at an arbitrary angle between their axis and the applied electric field, its electrophoretic mobility, $\mu_{\text {avg }}$, averaged over a random distribution of orientation is given by the following expression

$$
\mu_{\text {avg }}=\frac{\mu_{\|}+2 \mu_{\perp}}{3}
$$

In eq. (23), $\mu_{\|}$represents the electrophoretic mobility for filaments oriented parallel to an applied electric field, which can be calculated using the Smoluchowski's equation (24).

$$
\mu_{\|}=\frac{\varepsilon_{r} \varepsilon_{o} \zeta}{\eta}
$$

where $\varepsilon_{r}$ is the relative permittivity, $\varepsilon_{o}$ is the permittivity of a vacuum, and $\eta$ the solvent viscosity. While $\mu_{\perp}$ is the electrophoretic mobility for filaments oriented perpendicular to an applied electric field. In this case, Oshima included a relaxation effect correction to Henry's approach[31], leading to the following expression for $\mu_{\perp}$

$$
\mu_{\perp}=\frac{2 \varepsilon_{r} \varepsilon_{o} \zeta}{3 \eta}\left[f_{1}(\kappa a)-\left(\frac{z e \zeta}{k_{B} T}\right)^{2}\left\{f_{3}(\kappa a)+\left(\frac{m_{+}+m_{-}}{2}\right) f_{4}(\kappa a)\right\}\right]
$$

where $f_{1}(\kappa a)=\frac{3}{4}\left[1+\frac{1}{(1+2.55 /[\kappa a\{1+\exp (-\kappa a)\}])^{2}}\right], f_{3}(\kappa a)=\frac{\kappa a(\kappa a+0.162)}{2\left\{(\kappa a)^{3}+9.94(\kappa a)^{2}+18.7 \kappa a+0.147 \exp (-9.41 \kappa a)\right\}}$, $f_{4}(k a)=\frac{9 k a\{\kappa a+0.361 e x p(-0.475 \kappa a)+0.0878\}}{8\left\{(\kappa a)^{3}+10.8(\kappa a)^{2}+18.2 \kappa a+0.0633\right\}}$. In eq. (25), $z$ is the valence of counterions of the electrolyte solution, $e$ is the elementary electric charge, $m_{ \pm}=\frac{2 N_{A} \varepsilon_{r} \varepsilon_{0} k_{B} T}{3 \eta z \Lambda_{ \pm}^{0}}$ is the dimensionless ionic drag coefficient, and $\Lambda_{ \pm}^{0}$ are the ionic conductance for $\mathrm{K}^{+}$and $\mathrm{Cl}^{-}$species.

\section{Parameters calculation}

The values for the set of unknown parameters $\lambda_{-}, a, q, b i$, and $L_{p}$ usually depend on the specific electrolyte conditions, polymerization buffers, and sample preparation protocols, among other factors[8]. In this work, we found optimal values for these parameters that better reproduce the values for $\gamma_{0}, g_{1}\left(k_{s}, t\right), \mu_{\exp }$ and $D_{\exp }$ measured in the ELS and DLS experiments with $k_{s}=2.6354 \cdot 10^{7} / \mathrm{m}$ when $\theta=173^{\circ}$ and $\lambda=633 \mathrm{~nm}$.

In the first step, we used eq. (21) and the linear fit function for $\ln \left[g_{1}\left(k_{s}, t\right)\right]$ in Mathematica software v12.2 to determine the filament radius ' $a$ '. Meanwhile, substituting this parameter into eq. (22) and the use of the nonlinear fit function for

$$
-\frac{\Gamma(1 / 4)}{3 \pi}\left[\frac{k_{B} T}{4 \pi \eta}\left(\frac{5}{6}-\ln \left(k_{s} 2 a\right)\right)\right]^{3 / 4} \frac{k_{s}^{2} t^{3 / 4}}{L_{p}^{1 / 4}}
$$

in Mathematica software v12.2 yields the value for the persistence length $L_{p}$ [12].

In the second step, we use Mathematica software v12.2 and non-linear constrained global optimization techniques[32] to minimize the square sum cost function 


$$
F\left(\lambda_{+}, \lambda_{-}, a, q, L_{p}, b i\right)=\frac{\left(\mu_{a v g}\left(\lambda_{+}, \lambda_{-}, a, q, L_{p}, b i\right)-\mu_{e x p}\right)^{2}}{\mu_{\text {exp }}^{2}}+\frac{\left(D_{a v g}\left(\lambda_{+}, \lambda_{-}, a, L_{p}, b i\right)-D_{e x p}\right)^{2}}{D_{\text {exp }}^{2}}
$$

with respect to the set of parameters $\lambda_{-}, b i$, and $q$. We found that the algorithm "NMinimize" and the configuration:

$$
\begin{gathered}
\text { method }->\{\text { Randomsearch, serachpoints }->50, \text { Random Seed }->1, \\
\text { method }->\text { interior Point }\},\left\{-1 * 10^{-19} \mathrm{C}>q>-12 * 10^{-19} \mathrm{C},\right. \\
\left.10 / \mu m>\lambda_{-}>0.15 / \mu m\right\}, \text { accuracygoal }->8
\end{gathered}
$$

provided the most accurate and efficient minimization approach. We used the random search algorithm, which generates a population of random starting points and uses a local optimization method to converge to a local minimum. Then, the best local minimum is chosen to be the solution. We used 5,10,25,50 and larger numbers of search points. We found that numbers of search points larger than 50 generated the same optimal values. Further, we used the nonlinear interior point method, one of the most powerful algorithms to find the local minimum of a sum of squares[33]. Additionally, we used the numbers $(0,1,5,10)$ for the RamdonSeed parameter to consider different starting values in the random number generator algorithm. We found that the optimal values did not depend on these numbers. We constrained a range in the values of the parameters to avoid those with unphysical meaning and bracketed those typical values found in the literature. We used the "ParallelSum" and "RemoteKernel" Mathematica functions to run the Mathematica notebook on a computer cluster with 44 cores and 140Gb RAM.

\section{Experimental Section}

\subsection{Sample preparation}

Actin from rabbit skeletal muscle (> 99\% pure) was purchased from Cytoskeleton Inc. and used without further purification. We prepared three actin filament samples using the G-actin buffers, polymerization buffers, and electrolyte solutions tabulated in tables 1, 2, and 3 , respectively. We used the same sample preparation protocol for each sample. A $1.0 \mathrm{mg}$ of actin powder was reconstituted to $10 \mathrm{mg} / \mathrm{ml}$ G-actin density by adding $100 \mu L$ of Ultra-pure Distilled water Molecular Biology. Next, we added $2.40 \mathrm{~mL}$ of G-actin buffers (see table 1), aliquoted into experimental samples, and stored in cryo-tube vials at $-70^{\circ} C$. The G-actin solutions were incubated on ice for one hour to de-polymerize actin oligomers that may be formed during storage before polymerization. $20 \mu \mathrm{L}$ of polymerization buffers (see table 2) were added to $200 \mu \mathrm{L}$ G-actin solutions and transferred into Beckman coulter centrifuge tubes for one hour at room temperature to finish the polymerization stage. By balancing the needs of sample preservation and rapid run time, we centrifuge each experimental sample for 2 hours using the Allegra 64R Benchtop Centrifuge (Beckham Coulter) at $4^{\circ} \mathrm{C}$ using a speed of $50,000 \mathrm{G}$-force. Following this process, $22 \mu \mathrm{L}$ of protein 
pellet was obtained by extracting $198 \mu \mathrm{L}$ of the un-wanted supernatant $(90 \%)$. Consequently, we added $978.0 \mu \mathrm{L}$ of electrolyte solution (see table 3) to the pellet leading to a final volume of $1.0 \mathrm{~mL}$, and stored the final solution at $4^{\circ} \mathrm{C}$ overnight to achieve polymerization equilibrium in our samples. The pipetting tips used in our experiments were cut to an average diameter of $\sim 5-7 \mathrm{~mm}$ and prevent filament breakage[3], [9]. The $\mathrm{pH}$ of G-actin buffer, polymerization buffer, and electrolyte solutions were adjusted by adding either Hydrochloric acid $(\mathrm{HCl})$ volumetric standard or Sodium Hydroxide $(\mathrm{NaOH})$, pellets $97+\%$, A.C.S. reagents from Sigma Aldrich. The $\mathrm{pH}$ was measured with an accuracy of \pm 0.002 using Thermo scientific Orion Star ${ }^{\mathrm{TM}}$ A211 Benchtop pH Meter. Further details on this protocol are provided in the supplemental information to assure reproducibility.

\begin{tabular}{cccc}
\hline & Buffer 1 & Buffer 2 & Buffer 3 \\
\hline \hline Tris Base & $2 \mathrm{mM}$ & $2 \mathrm{mM}$ & $2 \mathrm{mM}$ \\
\hline $\mathrm{CaCl}_{2}$ & $0.2 \mathrm{mM}$ & $0.2 \mathrm{mM}$ & $0.2 \mathrm{mM}$ \\
\hline BME (Beta-Mercaptoethanol) & - & $0.1 \mathrm{mM}$ & - \\
\hline ATP (Adenosine triphosphate) & $0.5 \mathrm{mM}$ & $0.2 \mathrm{mM}$ & $0.5 \mathrm{mM}$ \\
\hline DTT (Dithiothreitol) & $0.2 \mathrm{mM}$ & - & $0.5 \mathrm{mM}$ \\
\hline $\mathrm{pH}$ & 7.80 & 7.66 & 8.23 \\
\hline Ionic Strength & 0.0057 & 0.0037 & 0.006 \\
\hline
\end{tabular}

Table 1: Table for G-actin buffers

\begin{tabular}{cccc}
\hline & Buffer 1 & Buffer 2 & Buffer 3 \\
\hline \hline $\mathrm{KCl}$ & $150 \mathrm{mM}$ & $150 \mathrm{mM}$ & $50 \mathrm{mM}$ \\
\hline $\mathrm{MgCl}_{2}$ & $2 \mathrm{mM}$ & $2 \mathrm{mM}$ & $2 \mathrm{mM}$ \\
\hline $\mathrm{pH}$ & 7.56 & 7.64 & 8.07 \\
\hline lonic strength & 0.155 & 0.155 & 0.055 \\
\hline
\end{tabular}

Table 2: Table for Polymerization Buffers

\begin{tabular}{cccc}
\hline & Buffer 1 & Buffer 2 & Buffer 3 \\
\hline \hline $\mathrm{KCl}$ & $0.1 \mathrm{M}$ & $0.1 \mathrm{M}$ & $0.1 \mathrm{M}$ \\
\hline $\mathrm{pH}$ & 7.72 & 7.66 & 8.06 \\
\hline lonic strength & 0.1 & 0.1 & 0.1 \\
\hline
\end{tabular}

Table 3: Table for Electrolytes 


\subsection{Light Scattering Experiments}

We used a Malvern ULTRA Zetasizer instrument equipped with a He-Ne $633 \mathrm{~nm}$ laser to measure actin filament's translational diffusion coefficient and electrophoretic mobility at low concentrations. The experiments were configured, and the data was recorded and analyzed using Zetasizer Xplorer software. The ULTRA Zetasizer features the Adaptive Correlation algorithm that uses information from the sample to determine how long it should be measured and the collection time that ensures data consistency. This feature also applies intelligent logic to separate erroneous data associated with transient artifacts such as dust or aggregates. Adaptive Correlation intelligently identifies rogue large particles and filters these from the presented data but retains consistently present populations. In all our measurements, we used 180 seconds as the equilibration time to thermally stabilize the sample at the desired temperature of $25^{\circ} \mathrm{C}$. The Zetasizer instrument uses a cell compartment that keeps the temperature constant during scattering measurements. Additionally, the attenuation factor was set to automatic. The attenuator uses 11 positions to control the beam intensity from $100 \%$ to $0.0003 \%$ in this mode. The instrument showed an attenuation factor between 10 and 11 across all measurements during our DLS and ELS experiments. We also selected "protein" for the material option with a refractive index of 1.450 and absorption of 0.001 . Furthermore, we selected "water" as the dispersant option with a refractive index of 1.33 , and a viscosity of $0.8872 \mathrm{mPa}$.s. It is essential to mention that the refractive index and absorption of the material have no bearing on the Z-average, polydispersity, and intensity distribution results.

In the DLS experiments, $1.0 \mathrm{ml}$ of actin filament solution was collected in the $12 \mathrm{~mm}$ square Polystyrene cuvette (DTS0012). The correlation functions were measured at the Back-scattering angle $\left(173^{\circ}\right)$, where the incident beam does not have to travel through the entire sample, and the effect of multiple scattering and dust is greatly reduced. We ran five consecutive, independent experiments for each actin filament sample to reduce statistical errors in the translational diffusion coefficient values. We calculated the average of the three longitudinal diffusion coefficient values with the lowest standard deviation and better correlation function to minimize error and increase reproducibility. The measurement duration was automatically determined from the detected count rate. In this mode, the lower the count rate, the longer the measurement duration, and the higher the noise. We used the 'General Purpose' analysis model, which uses a non-negative least squares (NNLS) analysis. It is the more suitable model for our case due to the unknown distribution of the size.

In the ELS experiments, we used the Malvern Panalytical Universal dip cell kit (ZEN1002) and the $12 \mathrm{~mm}$ square Polystyrene cuvette (DTS0012) for measuring the electrophoretic mobility of the actin filaments. We ran three independent experiments for each actin filament sample to reduce statistical errors in electrophoretic mobility values. The voltage selection and measurement process were set to automatic. The Zetasizer Xplorer software automatically measures the sample electrical conductivity in this mode. It adjusts the cell voltage to keep a low current flowing, close to $5 \mathrm{mS} / \mathrm{cm}$, in the sample. Otherwise, the sample temperature may increase near the electrodes, inducing bubble formation, sample degradation and, consequently, misleading the data measurements. The software automatically selects the most appropriate analysis and collection data model based on 
the cell type chosen, dispersant properties, and the sample's conductivity. We focused on the fast field reversal (FFR) of the phase analysis light scattering (see figure 6 ) since the mobility measured during this period is due to the electrophoresis of the particles only. It is not affected by electro-osmosis associated with the soft field reversal (SFR).

We improved reproducibility by including a pause between consecutive measurements. A time delay also helped reduce sample heating, allowing the sample to recover $25^{\circ} \mathrm{C}$ between consecutive measurements, reducing critical sample's degradation, and avoiding increasing mobility with sequential measurements. The minimum and maximum repeat runs per experiment were manually set to 10 and 30 , while the pause duration and pause between repeats were set to 10 and 60 seconds, respectively.

\section{Experimental Results}

The translational diffusion coefficients and polydispersity index (PDI) are obtained from the correlation function of the scattered intensity. The plot is depicted in figure (5). The average diffusion coefficient values with average standard deviation and percent error are summarized in the table (4). The diffusion coefficients of actin filaments were obtained at different $\mathrm{pH}$, reducing agents, ionic strength, and ATP concentration. All correlation plot results and intercepts were lower than one and within a range in polydispersity index of 0.3 - 0.5, indicating a good quality in our samples since the index range is low. Additionally, all our experimental size distribution measurements had a derived count rate higher than the minimum value of $100 \mathrm{kpcs}$ required to obtain a suitable measurement.

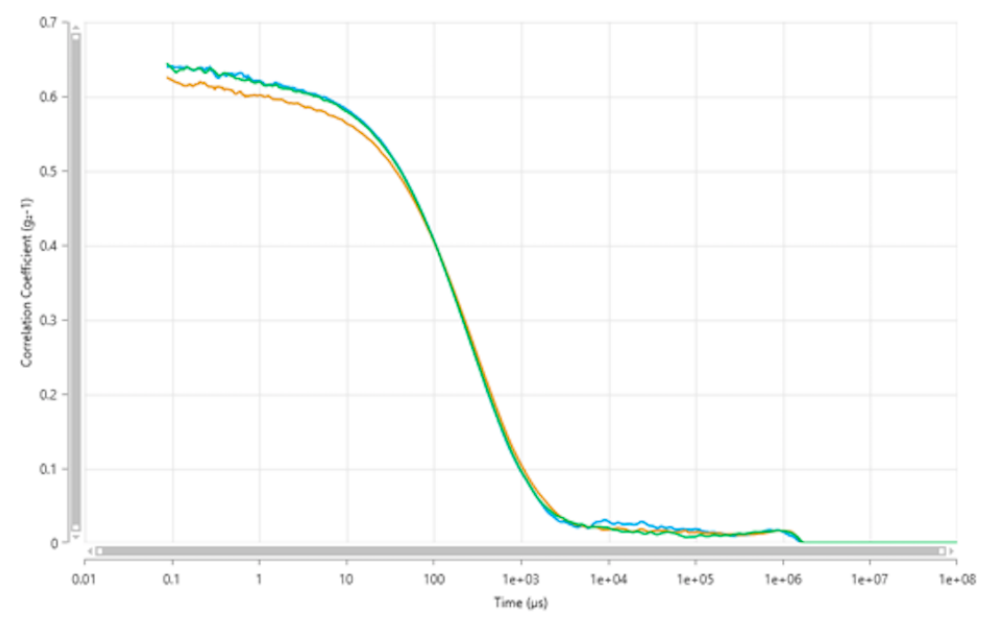

Figure 5: Correlation plot. Z-average of $190 \mathrm{~nm} \pm 8.3 \mathrm{~nm}$, polydispersity index (PDI) of 0.56 , derived mean count rate $366 \pm 15.7 \mathrm{kpcs}$, and a diffusion coefficient of $2.60 \pm$ $0.111 \mu \mathrm{m}^{2} / \mathrm{s}$.

The values obtained for the average electrophoretic mobility with standard deviation and percent error are tabulated in the table (5). An illustration on the graph for the electrophoretic mobility using buffer \#3 is shown in the figure (6). 


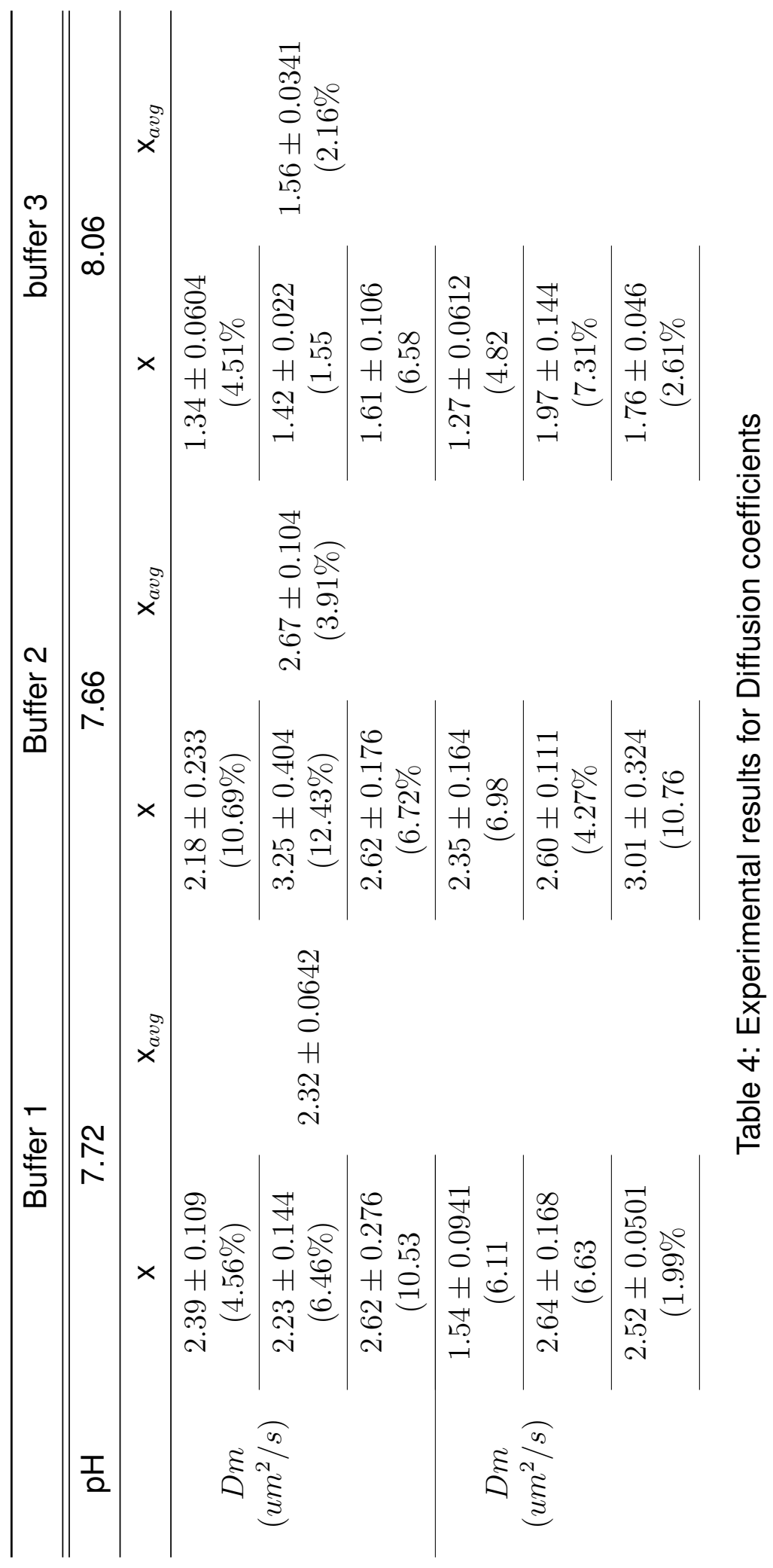


The quality factor is a parameter that derives from the phase analysis during the FFR stage of the measurement. Its value indicates the data quality. All our experimental electrophoretic mobility results had a quality factor within the range of $1.08-1.37$. These values are more significant than the minimum value of 1 required to get good data quality. Another evidence of good data quality is provided in the frequency shift plots obtained from our experimental results since there are no traces of noise, and the plots match very well. 


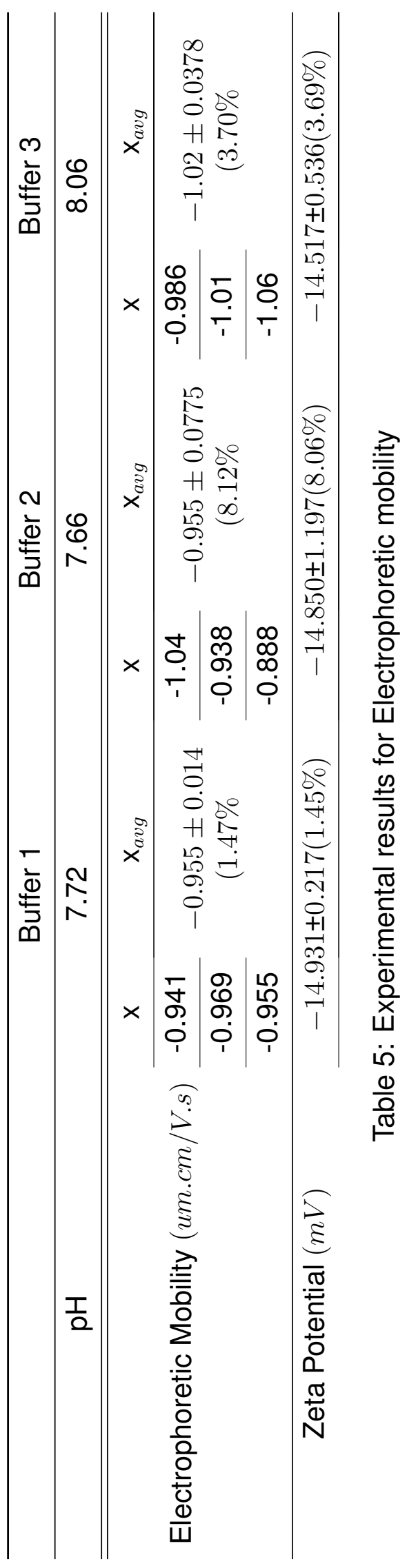



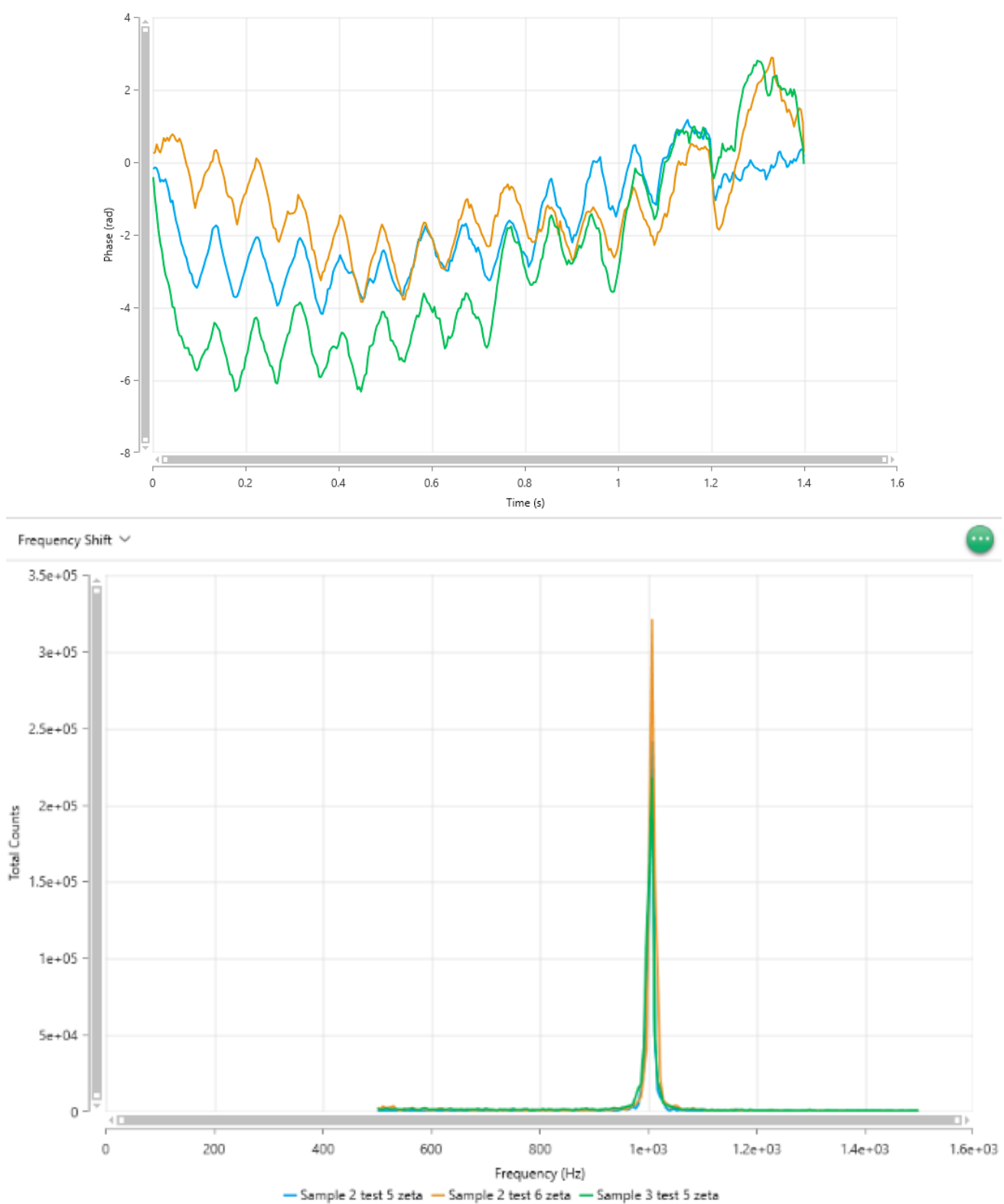

Figure 6: phase plot and frequency plot for buffer 3 with quality factor $1.37 \pm 0.321$, zeta potential $(\mathrm{mV})$ of $-13 \pm 0.506$, conductivity $(\mathrm{mS} \mathrm{cm}-1)$ of $12.4 \pm 1.69$, mobility $(\mu \mathrm{m} \cdot \mathrm{cm} / \mathrm{V} \cdot \mathrm{s}$ ) of $-1.02 \pm 0.0395$.

Using the correlation plot data of six independent sets of three runs each per buffer (see figure 4) and, and the fitting approach described in the section "Parameter calculation," we obtained the following results for the initial rate decay, the filament radius, and the persistence length $L_{p}$ for each buffer solution. 


\begin{tabular}{|c|c|c|c|}
\hline Buffer & $\gamma_{0}[1 / \mathrm{s}]$ & $d[\mathrm{~nm}]$ & $L_{p}[\mathrm{um}]$ \\
\hline \hline $\mathbf{1}$ & $6056.74 \pm 46.31$ & $10.77 \pm 0.168$ & $0.632 \pm 0.00376$ \\
\hline $\mathbf{2}$ & $5883.99 \pm 66.99$ & $11.27 \pm 0.263$ & $0.633 \pm 0.00797$ \\
\hline $\mathbf{3}$ & $3479.51 \pm 33.98$ & $26.29 \pm 0.306$ & $0.817 \pm 0.00676$ \\
\hline
\end{tabular}

Table 6: Calculations of $d, L_{p}$, and $\gamma_{0}$ from equations 21 and 22

\begin{tabular}{|c|c|c|c|}
\hline & Buffer \# 1 & Buffer \# 2 & Buffer \# 3 \\
\hline \hline$\lambda_{-}[1 / \mu m]$ & 1.676 & 2.119 & 1.234 \\
\hline $1 / \lambda_{-}[\mu m]$ & 0.5966 & 0.472 & 0.810 \\
\hline$a[n m]$ & 5.385 & 5.635 & 13.145 \\
\hline$q[e]$ & -3.428 & -3.653 & -8.275 \\
\hline$L_{p}[\mu m]$ & 0.632 & 0.633 & 0.817 \\
\hline$b$ & 0.7378 & 0.504 & 0.519 \\
\hline
\end{tabular}

Table 7: Optimal parameters

\section{Theoretical Results}

The optimal values for the parameters $\lambda_{-}, b$ and $q$ are tabulated in table 7. The values for $\lambda_{-}, \lambda_{+}$and $b$ were used to calculate the weight length $L_{w}=\frac{\sum_{N_{i}}\left(\frac{N_{i}}{m d}\right)^{2} Y\left(\frac{N_{i}}{m d}, \lambda_{+}, \lambda_{-}, b\right)}{\sum_{N_{i}}\left(\frac{N_{i}}{m d}\right) Y\left(\frac{N_{i}}{m d}, \lambda_{+}, \lambda_{-}, b\right)}$ and number length $L_{n}=\frac{\sum_{N_{i}}\left(\frac{N_{i}}{m d}\right) Y\left(\frac{N_{i}}{m d}, \lambda_{+}, \lambda_{-}, b\right)}{\sum_{N_{i}} Y\left(\frac{N_{i}}{m d}, \lambda_{+}, \lambda_{-}, b\right)}$ averages. We also calculated the polydispersity number $P D I=L_{n} / L_{w}$, the average hydrodynamic radius $R_{h}^{\exp }=\frac{k_{B} T}{6 \pi \eta D_{\text {exp }}}$, and the length distribution $Y\left(L, \lambda_{+}, \lambda_{-}, b\right)$. The values for the parameters $\lambda_{+}, R_{h}^{a v}, P D I, L_{n}$, and $L_{w}$ are given in table 8.

The filament length distributions $Y\left(L, \lambda_{+}, \lambda_{-}, b\right)$ are shown in Figure 7.

\begin{tabular}{|c|c|c|c|}
\hline & Buffer \# 1 & Buffer \# 2 & Buffer \# 3 \\
\hline \hline$\lambda_{+}[1 / \mu \mathrm{m}]$ & 16.76 & 21.19 & 12.34 \\
\hline$R_{h}^{a v}[\mu \mathrm{m}]$ & 0.0962 & 0.0922 & 0.157 \\
\hline$P D I$ & 0.562 & 0.591 & 0.548 \\
\hline$L_{n}[\mu \mathrm{m}]$ & 0.575 & 0.478 & 0.683 \\
\hline$L_{w}[\mu \mathrm{m}]$ & 0.986 & 0.809 & 1.246 \\
\hline
\end{tabular}

Table 8: Calculations using the optimal parameters 


\begin{tabular}{|c|c|c|c|}
\hline & Buffer \# 1 & Buffer \# 2 & Buffer \# 3 \\
\hline \hline Radius of Gyration, $R_{g}[\mu m]$ & 0.319 & 0.296 & 0.461 \\
\hline$R_{g, r o d}[\mu m]$ & 0.408 & 0.369 & 0.597 \\
\hline Polarizability, $<\alpha>^{1 / 3}[\mu m]$ & 0.528 & 0.484 & 0.765 \\
\hline$<\alpha>_{\text {rod }}^{1 / 3}[\mu m]$ & 0.662 & 0.593 & 0.949 \\
\hline
\end{tabular}

Table 9: Parameters were calculated from equations (6) and (9).

\section{Discussion}

\subsection{Length Distribution}

Our results for the weight- and the number-average length $L_{w}$ and $L_{n}$ varied for all cases, however the polydispersity index ratio didn't change. This is in agreement with previous work. Based on the structure and mobility of actin filaments, Janmey's group[9] observed a formation of long filaments in the F-actin's length distribution as they increased the actin and gelsolin molar ratios. Burlacu's group[25], used electron micrographs to analyze the length distribution of actin filaments under the presence of phalloidin-A, and IATRactin. They obtained higher values for these parameters; however, the PDI were similar across all cases. We noticed that the increase in the polydimerization growth rate of filaments for buffer \#1 led to an increase on $L_{n}$ and $L_{w}$ compared to the results obtained for buffer \#2. These differences may be, in part, due to the chemical composition of the two buffers. One difference between these two buffers is the 1.4-Dithiothreitol (DTT) and Beta-Mercaptoethanol (BME) concentrations. Buffer \#1 has $0.2 \mathrm{mM}$ DTT reducing agent, whereas buffer\# 2 contains $0.1 \mathrm{mM}$ BME. These two reducing agents are essential to prevent the formation of oligomers and agglomeration of monomers and maximize the availability of free G-actin monomers for polymerization[34]. Further, DTT has higher efficiency than BME in lowering F-actin's storage modulus, e.g. the overall resistance to deformation. Thus, the DTT concentration used in buffer \#1 could partially compensate and generate effects similar to the BME concentration used in buffer \#2. Other difference is that buffer \#1 has 2.5 times higher ATP concentration than buffer \#2. While the actin addition (elongation) rate depends on free ATP-G-actin concentration, the subunit loss rate does not [5], [35]. Although buffer \#1's growth association rates are higher than buffer \#2's by $21 \%$, the polydispersity index (PDI) values for buffer \#1 and \#2, remain similar. Additionally, the shearing parameter $b$, which is associated to the breakage fractioning of actin filaments in solution, are higher for buffer \#1 than buffer \#2 (see figure 7). We correlate this result to an increase in actin filament's lengths. Since the longer the filaments grow, the more filaments are exposed to shear and break.

Interestingly, buffer \#3 revealed the formation of much longer filaments compared to the other buffers, leading to an increase in the association rates (see table 7). Buffer \#3's association rates differ from buffer \#1 and \#2 by $26.35 \%$ and $41.73 \%$, respectively. An essential difference in the chemical composition is the 2.5 times higher DTT concentration of buffer \#3 compared to buffer \#1. We correlate the DTT's efficiency and increment in concentration to an increase in actin filament's length formation; therefore, leading to an 
increase in elongation rates. In addition, the increase in ATP concentration in the system, leads to an increment of free ATP-G-Actin free monomers in the solution. Thus, buffer \#3's $L_{n}$ and $L_{w}$ parameters result in higher values than the other two buffers (see figure 7). The shearing parameter for buffer \#3 resembles buffer \#2's result; however, buffer \#2 does not contain dithiothreitol in its chemical composition. We conclude that there is an inverse proportionality correlation between the shearing parameter and the DTT concentration in solution.

\subsection{Structural Parameters}

We evaluated 18 experimental autocorrelation plots (see figure 5) obtained from dynamic light scattering measurements using the dynamic structure factor theory. We extracted the initial decay rate, $\gamma_{0}$ in a time window ranging $\sim 10^{-6}$ to $3 \times 10^{-5}$ seconds to obtain the effective diameter $d$. Finally, we extracted the values for the persistence lengths, $L_{p}$, using equation (22), which are in good agreement with previous experimental work[19], [36]. Buffer \#1 and buffer \#2's persistence lengths are $0.632 \mu \mathrm{m}$ and $0.633 \mu \mathrm{m}$, respectively. However, buffer \#3's persistence length is $22.64 \%$ longer than the corresponding values for the other two buffers. This difference is due to the increase in filament's length of buffer \#3 rising from the higher presence of free ATP-G-actin monomers in the solution. Compared to the values usually reported from electron micrographs, the lower values for the persistence length and average contour filament length obtained in this work arise from the significant difference in the association rates at the filament ends that shift to sub-micro lengths, the maximum of the length distribution. In contrast, the exponential decay of the tail of the length distribution can only be measured experimentally due to microscopy resolution limitations.

The effective filament diameters shown in tables 6 and 7 agree with previous work. However, they are more significant than those obtained from bare molecular structure filament models. The use of Cong molecular structure model[37] for 13 polymerized Gactin monomers (see figure 8a) and the approach described by Marucho's group[33] yield an average filament diameter of $d_{M S}=4.77 \mathrm{~nm}$. As a result, the difference between the effective and bare diameters is equal to $\Delta d=10.77 \mathrm{~nm}-4.77 \mathrm{~nm}=6.0 \mathrm{~nm}$ and $\triangle d=11.27 \mathrm{~nm}-4.77 \mathrm{~nm}=6.5 \mathrm{~nm}$ for buffers \#1 and \#2, respectively. The increase in diameter can be explained using the MacMillian-Mayer theory for highly charged colloidal cylinders in monovalent salt solutions[38]. The approach predicts that the effective filament diameter is equal to the summation of the bare diameter, $d_{M S}$, and the contribution, $\triangle d$, from the filament charge and the electrical double layer (EDL) surrounding its surface. In particular, the calculation for a rod-like cylinder with uniform linear charge density $\lambda=-4 e / n m$, and diameter $d_{M S}=4.77 \mathrm{~nm}$ immersed in $0.1 \mathrm{M}$ monovalent salt solution yields an increment in the size of $\triangle d=5.49 \mathrm{~nm}$, which is similar to the values obtained in our previous calculations. The effective (integrated) monomer charges presented in the table 7 are smaller than those charges (-12e) obtained from bare G-actin molecular structures. This is due to the charge attenuation coming from the electrostatic screen generated by the high accumulation of counterions around the filament surface.

Interestingly, the effective diameter of $26.29 \mathrm{~nm}$ obtained for buffer \#3 is larger than 


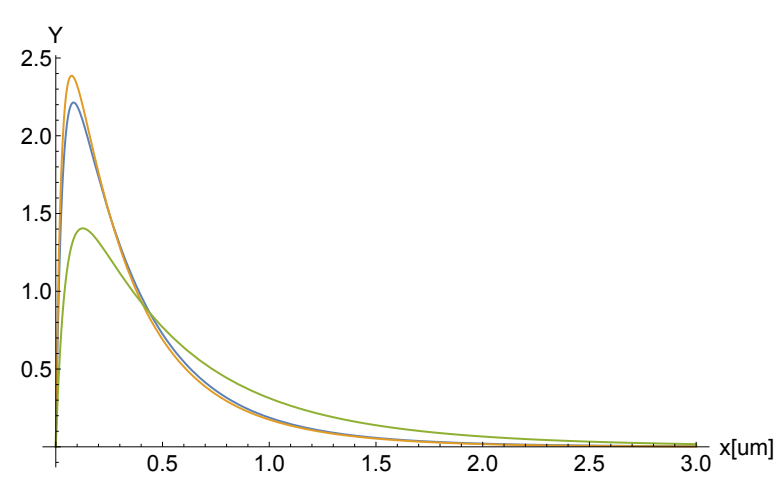

Figure 7: Filament length distribution $Y\left(\lambda_{+}, \lambda_{-}, L\right)$. Blue, orange, and green colors represent buffers \#1, \#2, and \#3 respectively.

those for buffers \#1 and \#2. We performed Transmission Electron Microscopy (TEM) experiments to get micrographs images on the three buffers (see the supplemental information). We observed the formation of single filaments for buffers \#1 and \#2; nevertheless, buffer \#3 shows not only the formation of single filaments but also the formation of actin filament bundles of different diameters. The combination of high ATP concentration, high DTT concentration, and low $\mathrm{KCl}$ concentration in the polymerization buffer \#3 could contribute to the formation of actin bundles. According to Lior Havir[39], an actin bundle's diameter has a minimum of three times thicker of a single filament's diameter, which is in agreement with our results for Buffer \#3. Additionally, the formation of longer filaments could lead to actin bundles in solution [26]. This assumption fits well our results since buffer \#3 produces longer actin filaments leading to smaller diffusion coefficients than buffers \#1 and \#2. Additionally, Tang's group [26] used different $\mathrm{KCl}$ concentrations of 30 $\mathrm{mM}, 50 \mathrm{mM}, 100 \mathrm{mM}$, and $150 \mathrm{mM}$ to induce actin bundles. They found out that actin bundles may form more easily at low concentrations of $\mathrm{KCl}$. This agrees with our results, since buffers \#1 and \#2 have $150 \mathrm{mM} \mathrm{KCl}$ concentration, whereas buffer \#3 has $50 \mathrm{mM}$ $\mathrm{KCl}$ only.

\subsection{Translational Diffusion Coefficient and Electrophoretic Mobility}

Quasielastic light scattering (QLS) experiments were performed by Janmey's group[9] to measure the translational diffusion coefficient of actin filaments using similar chemical compositions for the g-actin and polymerization buffers leading to diffusion coefficient results that agree with our results (see table 4). However, these values can significantly increase or decrease when considering different experimental protocols, chemical compounds, storage and preparation of actin monomers, polymerization, and techniques such as fluorescence photobleaching recovery (FPR), pyrene-labeled fluorescence, fluorescence and video microscopy[1]-[4], [6]. For instance, Wang's diffusion coefficient results differ from ours by 2-3 orders of magnitude because they measured the diffusion coefficient before the polymerization equilibrium is reached. In the same way, Kas's group[3] analyzed the diffusion coefficient through the tube model[40] and the concept of reptation[41] where a tube is embodied around a single filament. They used a much higher 


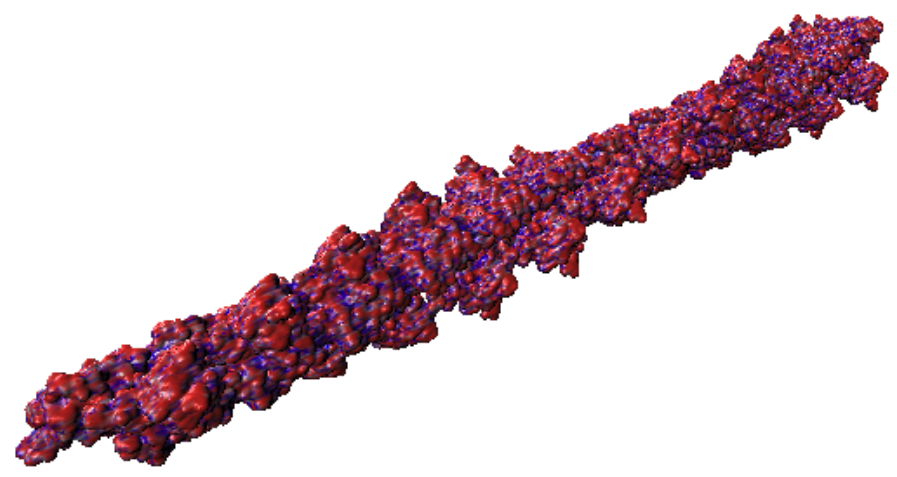

(a) F-actin

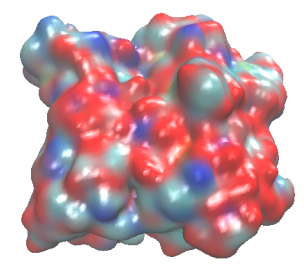

(b) G-actin

Figure 8: Molecular structure models

concentration of ATP and a concentration of actin up to three times larger than ours, generating longer filaments of about $\sim 20-50 \mu \mathrm{m}$ in length. As a result, they obtained an arithmetic mean of the diffusion coefficient, which is two orders of magnitude lower than our experimental results.

While our results for buffer \#1 and \#2 are quite similar, buffer \#3 showed a lower translational diffusion coefficient value of $1.56 \mu \mathrm{m}^{2} / \mathrm{s}$, which suggests that actin filaments are in average longer in buffer \#3. This is due to the high concentration of ATP and dithiothreitol (DTT) leading to more free ATP-G-actin monomers in the pool. Consequently, buffer \#3 leads to an increase in the associated growth rate of filaments. As the filaments increase in length, the translational diffusion coefficient decreases, which is in accordance with Zimmerle's results[42].

On the other hand, Takatsuki and Li[43], [44] have performed electrophoresis experiments using actin labeled with fluorescent dyes.Since they used buffers similar to ours, they have obtained an electrophoretic mobility value of $-0.85 \pm 0.07 \mu \mathrm{m} . \mathrm{cm} / \mathrm{V} . \mathrm{s}$, which is agreement with our experimental results (see table 5).

Overall, while our results for the longitudinal diffusion coefficient mainly depended on the length distribution, effective diameter, semiflexibility, chemical compounds, and reducing agents comprising G-actin buffers, the electrophoretic mobility mainly depended on the effective filament charge, the $\mathrm{pH}$ level, and the ionic strength.

\subsection{Other Properties}

According to Steinmetz's group[45], a comparison of Ca-G-actin, EGTA-G-actin, and Mg$\mathrm{G}$-actin polymerized with $100 \mathrm{mM} \mathrm{KCl}$ was studied to establish the impact of phalloidin over actin in a 2:1 molar concentration. The experimental protocols and buffers differ from ours by the sole presence of imidazole, NaN3, and EGTA. Similarly, De La Cruz's research is based on the structure of nucleotide-free actin filaments [46]. They found a radius of gyration ranging $\sim 2.4-2.5 \mathrm{~nm}$ in the presence of phalloidin, which is the same 
order of magnitude as our results (see table 9).

We also calculated the radius of gyration and the polarizability which depend on many factors such as the association rates, effective diameter, length distribution, persistence length, and the shearing parameter. Buffer \#1's parameters are $8.48 \%$ higher than buffer\#2's due to the increase in association rates associated with an increment of free ATP-G-actin monomers in the system; and consequently, an increase of the length distribution. Moreover, the fractioning of filaments in buffer \#1 is $\sim 32 \%$ higher than buffer \#2,leading to a contributing factor in this difference. In the same way, association rates, length distribution, and effective diameter in buffer \#3 are much higher than the other two buffers since we obtained longer filaments and the formation of actin filament bundles in the system. Consequently, these parameters increased by $30 \%$ and $36 \%$ compared to buffer \#1 and \#2, respectively.

We also analyzed the $R_{g, r o d}$ and $\langle\alpha\rangle_{\text {rod }}$ parameters (see table 9) for the rigid-rod case to understand the relevance of persistence length, $L_{p}$ in our calculations. Buffer \#1's rod values decrease $\sim 20 \%$, and buffer \#2's rigid-rod, $R_{g, r o d}$ and $\langle\alpha\rangle_{\text {rod }}$, decrease by $19.78 \%$ and $18.28 \%$, respectively, when considering a persistence length $L_{p}=0.63 \mu \mathrm{m}$. Whereas, buffer \#3's rod, $R_{\text {g,rod }}$ and $<\alpha>_{\text {rod }}$, decrease by $22.70 \%$ and $19.42 \%$, respectively, for a persistence length $L_{p}=0.82 \mu \mathrm{m}$. We concluded that the effect of persistence length on these parameters accounts for 20\% leading to a semiflexible case.

\section{Conclusions}

In this article, we introduced a unique approach that combines light scattering experiments and optimized theoretical approaches to characterize actin filaments' polyelectrolyte and hydrodynamic properties. We used Malvern ULTRA Zetasizer instrument to measure actin filament's translational diffusion coefficient and electrophoretic mobility at low protein concentration. We developed a novel sample preparation protocol based on bio-statistical tools to minimize errors and assure reproducibility in our results. This protocol was used for all the experiments. We considered three different buffers, g-actin and polymerization, used in previous works to elucidate the impact of their chemical composition, reducing agents, $\mathrm{pH}$ values, and ionic strengths on the filament properties.

Additionally, we used a novel multi-scale approach to calculate the translational diffusion coefficient and electrophoretic mobility of polydisperse actin filaments in aqueous salt solutions. The monodisperse translational diffusion coefficient calculations are based on the Stokes-Einstein formulation and a modified wormlike chain (WLC) model for the hydrodynamic radius. The monodisperse electrophoretic mobility calculations are carried out using a linear polymer representation of the WLC, which accounts for the balance between forces acting on each chain's monomer. This model and the Routine-Prager tensor for hydrodynamic interactions calculation are used to take the orientational average over all possible polymer conformations in the low electric field approximation. An asymmetric, exponential length distribution is used to characterize the actin filament polydispersity and the different increasing rate lengths of barbed and pointed ends. We used the length distribution to take the filament length average over the monodisperse translational diffusion coefficient and electrophoretic mobility expressions. The resulting expressions for 
the polydisperse translational diffusion coefficient and electrophoretic mobility depend on the persistence length, the effective filament diameter, the monomer charge, and the increasing rate length of barbed and pointed ends of the filaments. We considered typical experimental values for the degree of polymerization (370 G-actin proteins per um) and associate rates (barber end ten times larger than the pointed end). The values for the other parameters were adjusted to reproduce the experimental data obtained for three polymerization buffers. This characterization is novel since these parameter values are obtained from non-invasive experiments, and using the same experimental and hydrodynamic conditions.

Although buffers \#1 and \#2 produced some similar polyelectrolyte and hydrodynamic properties of actin filaments, there are many parameters that accounted for the theoretical differences, such as the elongation rates. Nevertheless, the results for buffer \#3 displayed substantial differences on the actin structural conformations. Compared to those values obtained from molecular structure models, our results revealed a lower value of the effective G-actin charge and a more significant value of the effective filament diameter due to the formation of the double layer of the electrolyte surrounding the filaments. Additionally, compared to the values usually reported from electron micrographs, the lower values of our results for the persistence length and average contour filament length agrees with the significant difference in the association rates at the filament ends that shift to sub-micro lengths, the maximum of the length distribution.

The optimized models and theories obtained in this article can be used and extended to calculate other actin filament's properties, including stability, the intrinsic viscosity[9], molecular weight (Mark-Houwink exponential coefficient), the axial tension, and the elastic stretch modulus [47], and the force-extension associated with the growth in length or the compression on the filament's shrinkage[48].

\section{Acknowledgements}

This work was supported by the National Institutes of Health [grant number 1SC1GM127187]. We thanks Dr. Arturo Ponce for his support in the microscopy sample preparation and data analysis.

\section{References}

[1] F. Wang, "Ph dependence of actin self-assembly.", Biophys J, 1989.

[2] F. Lanni, "Detection and characterization of actin monomers, oligomers, and filaments in solution by measurement of fluorescence photobleaching recovery.", Biophys J, 1984.

[3] J. Kas, "F-actin, a model polymer for semiflexible chains in dilute, semidilute, and liquid crystalline solutions j. kas, h. strey,' j. x. tang, d. finger,r. ezzell, e. sackmann,t and p. a. janmey.", Biophysical Journal, 1996. 
[4] H. L., "Tracer diffusion in f-actin and ficoll mixtures. toward a model for cytoplasm.", Biophys J, 1990.

[5] P. TD, "Rate constants for the reactions of atp- and adp-actin with the ends of actin filaments.", J Cell Biol, 1986.

[6] C. Bonet, "Rapid formation and high diffusibility of actin-cofilin cofilaments at low ph.”, Eur J Biochem, 2000.

[7] A. H. Crevenna, "Electrostatics control actin filament nucleation and elongation kinetics,", 2013.

[8] J. Xu, "Mechanical properties of actin filament networks depend on preparation, polymerization conditions, and storage of actin monomers.", Biophys J, 1998.

[9] P. A. Janmey, "Structure and mobility of actin filaments as measured by quasielastic light scattering, viscometry, and electron microscopy (received for publication, february 5, 1986) paul a. janmey, joyce peetermansg, ken s. zaner, thomas p. stossel, and toyoichi tanaka.", J Biol Chem, vol. 261, pp. 8357-8362, 1986, ISSN: 00219258. DOI: $10.1016 / \mathrm{s} 0021-9258$ (19)83920-0.

[10] - "The mechanical properties of actin gels: Elastic modulus and filament motions by paul a janmey, december 1994 journal of biological chemistry 269(51):3250313.", J Biol Chem, vol. 269, pp. 32 503-32513, 1994, ISSN: 0021-9258. DOI: 10 . 1016/s0021-9258(18)31663-6.

[11] K. Kroy, "Dynamic scattering from solutions of semiflexible polymers", Physical Review, 1997.

[12] M. Tassieri, "The self-assembly, elasticity, and dynamics of cardiac thin filaments", Biophysical Journal, 2008.

[13] J. H. McDonald, Handbook of Biological Statistics, S. H. Publishing, Ed. 2008.

[14] W. JM, The Protein Protocols Handbook, Springer-Verlag, Ed. New York, LLC, 2009.

[15] S. M. H., "Spectrophotometric determination of protein concentration", Current Protocols in Food Analytical Chemistry, 2002.

[16] M. L. Mansfield, "Transport properties of rodlike particles.", Macromolecules, 2008.

[17] _ " "Transport properties of wormlike chains with applications to double helical dna and carbon nanotubes.", Macromolecules, 2008.

[18] A. R. VÃ $\mid$ lkel, "On the mobility of stiff polyelectrolytes.", The Journal of Chemical Physics, 1995.

[19] Y. Jeune-Smith, "Engineering the length distribution of microtubules polymerized in vitro.", Soft Matter, 2010.

[20] H. Yamakawa, "Translational friction coefficient of wormlike chains.", Macromolecules, 1973.

[21] W. P. J. T. Van Der Drift, "Electrophoretic mobility of a cylinder with high surface charge density.", Journal of Colloid and Interface Science, 1979. 
[22] J. G. Kirkwood, "The intrinsic viscosities and diffusion constants of flexible macromolecules in solution.", The Journal of Chemical Physics, 1948.

[23] M. Muthukumar, "Theory of electrophoretic mobility of polyelectrolyte chains.", Macromolecular Theory and Simulations, 1994.

[24] I.-C. Yeh, "Diffusion and electrophoretic mobility of single-stranded rna from molecular dynamics simulations.", Biophysical journal, 2004.

[25] S. Burlacu, "Distribution of actin filament lengths measured by fluorescence microscopy.", Am J Physiol, 1992.

[26] J. X. Tang, "The polyelectrolyte nature of f-actin and the mechanism of actin bundle formation", Journal of Biological Chemistry, 1996.

[27] D. D, "Elongation of actin filaments is a diffusion-limited reaction at the barbed end and is accelerated by inert macromolecules.", J Biol Chem., 1986.

[28] G. Schulz, "About the kinetics of the chain polymerizations", Journal of Physical Chemistry, 1939.

[29] R. Gotter., "Dynamic light scattering from semidilute actin solutions: A study of hydrodynamic screening, filament bending stiffness, and the effect of tropomyosin/troponinbinding", Macromolecules, 1996.

[30] H. Ohshima, "Approximate analytic expression for the electrophoretic mobility of moderately charged cylindrical colloidal particles", Langmuir, 2015.

[31] D. C. Henry, "The cataphoresis of suspended particles.", Proceedings of the Royal Society of London., 1931.

[32] S. Vergara-Perez, "Mpbec, a matlab program for biomolecular electrostatic calculations", Comput Phys Commun, 2016.

[33] M. Marucho, "A java application to characterize biomolecules and nanomaterials in electrolyte aqueous solutions.", Computer Physics Communications, 2019.

[34] Tang, "Thiol oxidation of actin produces dimers that enhance the elasticity of the f-actin network jay x. tang", 1999.

[35] D Pantaloni, "A model for actin polymerization and the kinetic effects of atp hydrolysis", 1985.

[36] Y. Ari, "Relationship between the flexibility and the motility of actin filaments: Effects of ph", Biochemical and Biophysical Research Communications, 2008.

[37] Y. Cong, "Crystallographic conformers of actin in a biologically active bundle of filaments.", Journal of Molecular Biology, 2008.

[38] D. Stigter, "Interactions of highly charged colloidal cylinders with applications to double-stranded dna", Biopolymers, 1977.

[39] L. Haviv, "Thickness distribution of actin bundles in vitro", Eur Biophys J, 2008.

[40] E. S. F., "The statistical mechanics of polymerized material", Proceedings of the Physical Society, 1967. 
[41] G. P.G.d., "Reptation of a polymeri chain in the presence of fixed obstacles", The Journal of Chemical Physics, 1971.

[42] C. T. Zimmerle, "Effect of ph on the mechanism of actin polymerization,", 1988.

[43] H. Takatsuki, "References electrophoretic mobility of nano-sized actin filaments in biomolecular device", 2006.

[44] G. Li, "Single filament electrophoresis of f-actin and filamentous virus fd,", 2005.

[45] M. O. Steinmetz, "A correlative analysis of actin filament assembly, structure, and dynamics", The Journal of Cell Biology, 1997.

[46] E. De La Cruz, "Polymerization and structure of nucleotide-free actin filaments", Journal of Molecular Biology, 2000.

[47] X. Liu, "Mechanics of f-actin characterized with microfabricated cantilevers", Biophysical Journal, 2002.

[48] J. S. Palmer, "Constitutive modeling of the stress-strain behavior of $\mathrm{f}$-actin filament networks", Science Direct, 2008.

[49] S. D.F., "The beer-lambert law", Journal of Chemical Education, 1962.

\section{Supplemental Information}

\section{Materials}

Protein Actin (>99\% pure) rabbit skeletal muscle $1 \mathrm{mg}$ was purchased from Cytoskeleton Inc and stored at $4^{\circ} \mathrm{C}$. For size and zeta potential measurements, disposable cells DTS0012 and dip cell ZEN1002 were purchased from Malvern. Ultra-pure DI water was purchased from Sigma-Aldrich and used in all buffer and protein preparation.

Instruments used

1. Ohaus Explorer Pro-Scale.

2. Vortex -2- Genie.

3. Beckman Coulter Allegra 64R Benchtop centrifuge machine.

4. Thermo Scientific Orion Star A211 Bench top pH meter.

5. Andrew Alliance electronic pipettes.

6. Malvern Panalytical Zetasizer Ultra. 


\section{Sample preparation}

Experiment lasts for a week. First day, prepare the salt aqueous solutions of $50 \mathrm{mM}$ $\mathrm{CaCl}_{2}, 1 \mathrm{M}$ of $\mathrm{KCl}, 0.1 \mathrm{M}$ of $\mathrm{KCl}$ and $50 \mathrm{mM}$ of $\mathrm{MgCl}_{2}$ to a desired volume of $10 \mathrm{ml}$ using ultra pure distilled Water and store the solution at $4^{\circ} \mathrm{C}$ for $24 \mathrm{hrs}$. To keep fresh stock, this process should be repeated on a weekly basis. Never use stock past a week. Second day, have ice ready in a cooler, this process will be done on ice. Prepare G- actin buffers and polymerization buffers with desired $\mathrm{pH}$ to a volume of $5 \mathrm{ml}$ as shown in the buffer tables. For all the buffers $\mathrm{pH}$ was adjusted by adding either Hydrochloric acid $(\mathrm{HCl})$ or sodium hydroxide $(\mathrm{NaOH})$ and measured using Thermo scientific Orion Star ${ }^{\mathrm{TM}}$ A211 Bench top $\mathrm{pH}$ Meter. Again store the buffers at $4^{\circ} \mathrm{C}$ for $24 \mathrm{hrs}$. ATP (Adenosine triphosphate) and DTT (Dithiothreitol) are initially stored on the fridge, when ready to prepare the solutions, wait about 5 min for ATP and DTT to thaw. Immediately after use, snap freeze with liquid Nitrogen and store back on the fridge. Third day, be prepared with ice in a cooler. Transfer $1 \mathrm{mg} \mathrm{G}$-actin protein solid powder (one unit from Cytoskeleton.inc) into a $3 \mathrm{~mL}$ glass vile. Reconstitute the protein to $10 \mathrm{mg} / \mathrm{ml}$ G-actin density using $100 \mathrm{uL}$ of ultra pure distilled Water and vortex the vile to dissolve the solid protein as much as you can. Add $2.4 \mathrm{~mL}$ G-Actin buffer(according to the $\mathrm{pH}$ ) into the vile containing the protein and also dissolve the aggregates as much as possible by vortexing at slow speed. Aliquot the protein solution into experimental sized amounts. Taking in consideration that muscle actin is a liable protein and should be handled with care, and also to avoid repeated free-thaw cycles, certain experimental sized amounts were considered according to how many experiments and how concentrated we are working with. We suggest to have 4 cryotubes containing $250 \mathrm{uL}$ each and stored the rest in one Cryotube vial. Snap-freeze the cryotube viles with liquid Nitrogen and store immediately in the freezing container at $-70^{\circ} \mathrm{C}$. Fourth day, be ready with ice in a cooler. Extract one cryotube vile containing 250 $\mathrm{uL}$ of protein solution and wait $5 \mathrm{~min}$ until the protein solution thaw. Incubate on ice for an hour to depolymerize actin oligomers that form during storage. Extract $200 \mathrm{uL}$ of protein solution and place into a microcentrifuge tube and add $20 \mathrm{uL}$ of polymerization buffer (1/10 th volume) according to the $\mathrm{pH}$. Vortex the solution at slow speed. Incubate at room temperature for one hour and turn off light to avoid any potential bacteria growth. Turn on the centrifuge machine 15 minutes before its usage. Set-up the centrifuge machine at 50,000 RCF (Relative Centrifugal Force) for 2 hours at $4^{\circ} \mathrm{C}$, and the acceleration and deceleration should be set at 2 each and Centrifuge the protein for 2 hours. After this process, remove the top $90 \%$ of supernatant $(198 \mathrm{uL}$ ) carefully, by extracting the protein solution from the centrifuge in the following manner ( $100 \mathrm{uL}, 50 \mathrm{uL}$, and $48 \mathrm{uL}$ ). You would have left $22 \mathrm{uL}$ of filaments. Set the pipette in titration mode and add $978 \mathrm{uL}$ of $0.1 \mathrm{M} \mathrm{KCl}$ (electrolyte) to the $22 \mathrm{uL}$ pallet according to the $\mathrm{pH}$ and place carefully at the fridge at $4^{\circ} \mathrm{C}$ and leave it overnight. All the buffers and solutions we are using is stored in the ice cooler during whole experiment. Last day, cut the pipette tips about $7 \mathrm{~mm}$ above from the tip so that we can collect most of the protein without breaking the filaments. Turn on the zeta sizer ultra system 10 minutes before its usage. Collect the protein $(1 \mathrm{ml})$ as much as you can by setting the pipette in titration mode and transfer the protein to a $12 \mathrm{~mm}$ square polystyrene cuvette (DTS0012) for size measurements and use dip cell (ZEN1002) in 
DTS0012 to measure electrophoretic mobility. Clean the dip cell thoroughly using ethanol and water after each use.

\section{Salt aqueous solution preparation}

\section{a. Preparation of $50 \mathrm{mM} \mathrm{CaCl} 2$}

1. Use the Ohaus Explorer Pro Scale to measure $55.50 \mathrm{mg}$ of dried powder, $97 \%$ crystalline, $\mathrm{CaCl} 2$.

2. Transfer the measured amount of $55.5 \mathrm{mg}$ of $\mathrm{CaCl} 2$ in a $15 \mathrm{ml}$ conical CORNING tube.

3. Add $10 \mathrm{ml}$ of Ultra-pure Distilled water Molecular Biology to dissolve the salt to make $50 \mathrm{mM}$ of $\mathrm{CaCl} 2$

4. Vortex the solution for 90 seconds at 5-7 speed using the Vortex -2- Genie.

5. Place the $15 \mathrm{ml}$ conical tube containing the solution in the bottom of the fridge at $4^{\circ} \mathrm{C}$.

\section{b. Repeat the process (a1- a5) and measure $745.5 \mathrm{mg}$ of $\mathrm{KCl}$ to make $1 \mathrm{M}$ of $\mathrm{KCl}$}

c. Repeat the process (a1 - a5) and measure $76.22 \mathrm{mg}$ of $\mathrm{KCl}$ to make $0.1 \mathrm{M}$ of $\mathrm{KCl}$

d. Repeat the process (a1 - a5) and measure $47.61 \mathrm{mg}$ of anhydrous, $99 \%$ crystalline, $\mathrm{MgCl} 2$, to make $50 \mathrm{mM}$ of $\mathrm{MgCl} 2$

Notes: Pour $40-45 \mathrm{ml}$ of Ultra pure distilled water into a $50 \mathrm{ml}$ conical CORNING tube. Complete step a3 by getting DI water from the $50 \mathrm{ml}$ tube. To keep fresh stock, this process should be repeated on a weekly basis. Never use stock past a week!

We prepared three different buffers. Each buffer has 3 samples and performed the experiments.Preparation of each buffer has given below.

\section{Buffer 1 with pH 7.72}

\subsection{G-actin buffer with pH 7.72}

1. Once the stock from the previous process is complete, proceed to the making of 5 $\mathrm{ml}$ G-Actin buffers.

2. Have ice ready in a cooler, this process will be done on ice.

3. To obtain a G-Actin buffer of $2 \mathrm{mM}$ Tris- $\mathrm{HCl} \mathrm{pH} 7.6,0.2 \mathrm{mM} \mathrm{CaCl}$, $0.5 \mathrm{mM}$ ATP (Adenosine triphosphate), $0.2 \mathrm{mM}$ DTT (Dithiothreitol), proceed to the following step. 
4. In a $15 \mathrm{ml}$ conical CORNING tube, add $4.845 \mathrm{ml}$ of Ultra-pure water, $100 \mathrm{uL}$ of Tris$\mathrm{HCl} \mathrm{pH} 7.6,20 \mathrm{uL}$ of $50 \mathrm{mM} \mathrm{CaCl} 2,10 \mathrm{uL}$ of $100 \mathrm{mM}$ DTT (Dithiothreitol), $25 \mathrm{ul}$ of $100 \mathrm{mM}$ ATP (Adenosine triphosphate).

5. Vortex the solution for 60 seconds at 5 speed using the Vortex-2-Genie f. Place the $15 \mathrm{~mL}$ conical tube containing the solution in the bottom of the fridge at $4^{\circ} \mathrm{C}$.

\subsection{Polymerization Buffer with 7.56}

1. Once the stock from the previous process is complete, proceed to the making of 5 $\mathrm{ml}$ Polymerization buffer.

2. Have ice ready in a cooler; this process will be done on ice.

3. To obtain a buffer of $150 \mathrm{mM} \mathrm{KCl}, 2 \mathrm{mM} \mathrm{MgCl}$, proceed to the following steps 4 and 5 for $\mathrm{pH} 7.56$.

4. In a $15 \mathrm{ml}$ conical CORNING tube, add $750 \mathrm{uL}$ of $1.0 \mathrm{M} \mathrm{KCl}, 200 \mathrm{uL}$ of $50 \mathrm{mM}$ $\mathrm{MgCl} 2$, and $4.05 \mathrm{ml}$ of Ultra-Pure water.

5. Vortex the solution for 60 seconds at speed 5 using the Vortex-2-Genie.

6. Place the $15 \mathrm{ml}$ conical tube containing the solution in the bottom of the fridge at $4^{\circ} \mathrm{C}$.

\subsection{Electrolyte}

$0.1 \mathrm{M} \mathrm{KCl}$ with $\mathrm{pH} 7.72$

\section{Buffer 2 with pH 7.66}

\subsection{G-actin buffer with $\mathrm{pH} 7.66$}

1. Once the stock from the previous process is complete, proceed to the making of 5 $\mathrm{ml}$ G-Actin buffers.

2. Have ice ready in a cooler, this process will be done on ice.

3. To obtain a G-Actin buffer of $2 \mathrm{mM}$ Tris- $\mathrm{HCl} \mathrm{pH} 7.6,0.2 \mathrm{mM} \mathrm{CaCl}, 0.2 \mathrm{mM}$ ATP (Adenosine triphosphate), $0.1 \mathrm{mM}$ Beta Mercaptoethanol, proceed to the following step.

4. In a $15 \mathrm{ml}$ conical CORNING tube, add $100 \mathrm{uL}$ of $100 \mathrm{mM}$ Tris- $\mathrm{HCl} \mathrm{pH} 7.6,20 \mathrm{uL}$ of $50 \mathrm{mM} \mathrm{CaCl}, 10 \mathrm{ul}$ of $100 \mathrm{mM}$ ATP (Adenosine triphosphate), $10 \mathrm{uL}$ of $50 \mathrm{mM}$ Beta-Mercaptoethanol and $4.86 \mathrm{ml}$ of Ultra-pure water.

5. Vortex the solution for 60 seconds at 5 speed using the Vortex-2-Genie. 
6. Place the $15 \mathrm{ml}$ conical tube containing the solution in the bottom of the fridge at $4^{\circ} \mathrm{C}$.

\subsection{Polymerization Buffer with 7.64}

1. Once the stock from the previous process is complete, proceed to the making of 5 $\mathrm{ml}$ Polymerization buffer.

2. Have ice ready in a cooler; this process will be done on ice.

3. To obtain a buffer of $150 \mathrm{mM} \mathrm{KCl,} 2 \mathrm{mM} \mathrm{MgCl}$, proceed to the following steps for $\mathrm{pH} 7.64$

4. In a $15 \mathrm{ml}$ conical CORNING tube, add $750 \mathrm{uL}$ of $1.0 \mathrm{M} \mathrm{KCl}, 200 \mathrm{uL}$ of $50 \mathrm{mM}$ $\mathrm{MgCl} 2$, and $4.05 \mathrm{ml}$ of Ultra-Pure water for $\mathrm{pH} 7.64$.

5. Vortex the solution for 60 seconds at speed 5 using the Vortex-2-Genie.

6. Place the $15 \mathrm{ml}$ conical tube containing the solution in the bottom of the fridge at $4^{\circ} \mathrm{C}$.

\subsection{Electrolyte}

$0.1 \mathrm{M} \mathrm{KCl}$ with $\mathrm{pH} 7.66$

\section{Buffer 3 with pH 8.06}

\subsection{G-actin buffer with pH 8.06}

1. Once the stock from the previous process is complete, proceed to the making of 5 $\mathrm{ml}$ G-Actin buffers.

2. Have ice ready in a cooler, this process will be done on ice.

3. To obtain a G-Actin buffer of $2 \mathrm{mM}$ Tris- $\mathrm{HCl} \mathrm{pH} 7.6,0.2 \mathrm{mM} \mathrm{CaCl}, 0.5 \mathrm{mM}$ ATP (Adenosine triphosphate), $0.5 \mathrm{mM}$ DTT(Dithiothreitol), proceed to the following step.

4. In a $15 \mathrm{ml}$ conical CORNING tube, add $100 \mathrm{uL}$ of $100 \mathrm{mM}$ Tris- $\mathrm{HCl} \mathrm{pH} 8,20 \mathrm{uL}$ of $50 \mathrm{mM} \mathrm{CaCl} 2,25 \mathrm{uL}$ of $100 \mathrm{mM}$ ATP (Adenosine triphosphate), $25 \mathrm{uL}$ of $100 \mathrm{mM}$ DTT(Dithiothreitol) and $4.83 \mathrm{~mL}$ of Ultra-pure water.

5. Vortex the solution for 60 seconds at 5 speed using the Vortex-2-Genie.

6. Place the $15 \mathrm{~mL}$ conical tube containing the solution in the bottom of the fridge at $4^{\circ} \mathrm{C}$. 


\subsection{Polymerization Buffer with $\mathbf{8 . 0 7}$}

There are three kinds of polymerization buffer with three different sets of $\mathrm{pH}$.

1. Once the stock from the previous process is complete, proceed to the making of 5 $\mathrm{mL}$ Polymerization buffer.

2. Have ice ready in a cooler; this process will be done on ice.

3. To obtain a buffer of $50 \mathrm{mM} \mathrm{KCl}, 2 \mathrm{mM} \mathrm{MgCl}$, proceed to the following step.

4. In a $15 \mathrm{~mL}$ conical CORNING tube, add $250 \mathrm{uL}$ of $1.0 \mathrm{M} \mathrm{KCl}, 200 \mathrm{uL}$ of $50 \mathrm{mM}$ $\mathrm{MgCl} 2$, and $4.55 \mathrm{~mL}$ of Ultra-Pure water for $\mathrm{pH} 8.07$.

5. Vortex the solution for 60 seconds at speed 5 using the Vortex-2-Genie.

6. Place the $15 \mathrm{~mL}$ conical tube containing the solution in the bottom of the fridge at $4^{\circ} \mathrm{C}$.

\subsection{Electrolyte}

$0.1 \mathrm{M} \mathrm{KCl}$ with 8.06

Note:Using the Orion Star A211, we measured the $\mathrm{pH}$ buffers of all samples.

\section{Protein Reconstitution}

1. Transfer $1 \mathrm{mg} \mathrm{G}$-actin protein solid powder (one unit from Cytoskeleton.inc) into a 3 $\mathrm{mL}$ glass vile.

2. Add $100 \mathrm{uL}$ of Ultra pure Distilled Water into the $3 \mathrm{~mL}$ glass vile to reconstitute 10 $\mathrm{mg} / \mathrm{ml} \mathrm{G}$-actin density.

3. Vortex the glass vile for $30-40$ seconds at speed 3 to dissolve the solid protein as much as you can.

4. Add $2.4 \mathrm{~mL} \mathrm{G}$-Actin buffer(according to the $\mathrm{pH}$ ) into the vile containing the protein.

5. Vortex the glass vile for $30-40$ seconds at speed 4 to dissolve the aggregates as much as you can.

6. Aliquot the protein solution into experimental sized amounts. Use a $1.8 \mathrm{~mL}$ Cryotube vial. We suggest to have 4 cryotubes containing $250 \mathrm{uL}$ each and stored the rest in one Cryotube vial.

7. Snap-freeze the cryotube viles with liquid Nitrogen (follow the notes for liquid nitrogen use) and store immediately in the Dr. Lorenzo Brancaleon's freezing container at $-80^{\circ} \mathrm{C}$. 
Note: Taking in consideration that muscle actin is a liable protein and should be handled with care, and also to avoid repeated free-thaw cycles, certain experimental sized amounts were considered according to how many experiments and how concentrated we are working with.

\section{Polymerization at dilute (low) actin concentration (use 3 tubes)}

1. Extract one cryotube vile containing $250 \mathrm{uL}$ of protein solution form the $-80^{\circ} \mathrm{C}$ fridge.

2. Wait 5 min until the protein solution thaw.

3. Incubate on ice for an hour to depolymerize actin oligomers that form during storage.

4. From the cryotube vial containing $250 \mathrm{uL}$, extract $200 \mathrm{uL}$ of protein solution and place into a microcentrifuge tube polypropylene (Beckman Coulter).

5. Add $20 \mathrm{uL}$ of polymerization buffer (1/10 th volume) according to the $\mathrm{pH}$, to the microcentrifuge tube containing $200 \mathrm{uL}$ protein solution.

6. Vortex the solution for $40-50$ seconds at speed 2-3 using the Vortex-2-Genie.

7. Incubate at room temperature for one hour. Turn off light to avoid any potential bacteria growth.

8. Turn on the Beckman coulter Allegra 64R Benchtop centrifuge machine 15 minutes before its usage.

9. Set-up the centrifuge machine at 50,000 RCF (Relative Centrifugal Force) for 2 hours at $4^{\circ} \mathrm{C}$, and the acceleration and deceleration should be set at 2 each.

10. Centrifuge the protein for 2 hours.

11. After this process, carefully extract the protein solution from the centrifuge in the following manner (100 uL, $50 \mathrm{uL}$, and $48 \mathrm{uL}$ ).

12. Remove the top $90 \%$ of supernatant (198 uL); you would have left $22 \mathrm{uL}$ of filaments.

13. Set the pipette in titration mode and add $978 \mathrm{uL}$ of $0.1 \mathrm{M} \mathrm{KCl}$ (electrolyte) to the 22 $\mathrm{uL}$ pallet according to the $\mathrm{pH}$.

14. Place carefully at the bottom fridge and leave it overnight.

Note: Take in consideration that after polymerization, the actin filaments are found to be at optimal condition for the next $24 \mathrm{hr}$. After this, actin filaments will start depolymerizing.

Note: ATP (Adenosine triphosphate) and DTT (Dithiothreitol) are initially stored on the fridge, when ready to prepare the solutions, wait about 5 min for ATP and DTT to thaw. Immediately after use, snap freeze with liquid Nitrogen and store back on the fridge. 
Obtain liquid Nitrogen from the Nitrogen tank located in Dr. Nash's lab. Place the liquid nitrogen in a half-bottom-cut bottle. It will evaporate fast so as soon as ATP is used, snap freeze it by placing the ATP container inside the liquid nitrogen for 10 seconds. After this process, place back in the fridge.

\section{Experimental set up}

1. Clean the scissor using ethanol and water.

2. Cut the pipette tips about $3 \mathrm{~mm}$ to $5 \mathrm{~mm}$ above from the tip so that we can collect the protein without breaking the filaments.

3. Set the pipette in titration mode and titrate the protein in $45^{\circ}$ angle mode.

4. Collect the protein as much as you can and transfer the protein to a $12 \mathrm{~mm}$ square Polystyrene cuvette (DTS0012) for size measurements and use Dip cell (ZEN1002) to measure Electrophoretic mobility.

5. Turn on the zeta sizer ultra system 10 minutes before its usage.

6. Size measurements and zeta potential measurements are taken by DLS method using Zeta sizer Ultra with ZS xplorer software.

\section{Protein Concentration}

We used the Pur-A-Lyzer Midi Dialysis Kit of 1000 molecular weight cut-off (MWCO) for 24 hours on the supernatant following Sigma-Aldrich protocols. After the 24 hours dialysis, we proceeded to transfer the protein solution into a $400 \mathrm{uL}$ quartz cuvette to measure the absorbance by using the UV-Vis Spectrophotometer[15]. We measured the absorbance in the range of 220 - $320 \mathrm{~nm}$ of three samples for each buffer. We used the 'SpectraGryph' to obtain the absorbance at $290 \mathrm{~nm}$. Once the absorbance was obtained, we proceeded to use Beer-Lambert's law[49] which combines parameters such as the molar extinction coefficient $\varepsilon$ and the absorbance $A$ of our sample to predict the protein concentration. We determined the protein concentration of the supernatant by absorbance at $290 \mathrm{~nm}$ using an extinction coefficient of $0.62 \mathrm{mg}^{-1} \mathrm{mlcm}^{-1}[1]$. This data and the total initial protein concentration were used to calculate the protein concentration in our experiments.

\subsection{Protein Dialysis Procedure}

1. Fill the Pur-A-Lyzer with $0.8 \mathrm{~mL}$ of ultrapure water; incubate for at least 5 minutes. Empty the tube.

2. Load sample into the Pur-A-Lyzer tube. Close the tube with the provided caps (do not apply force). 


\begin{tabular}{|c|c|c|c|c|c|c|}
\hline & Buffer 1 & Avg. & Buffer 2 & Avg. & Buffer 3 & Avg. \\
\hline \multirow{3}{*}{ Absorbance, A } & 0.02939 & \multirow{3}{*}{$\begin{array}{c}0.02822 \\
\pm 0.00174\end{array}$} & 0.009225 & \multirow{3}{*}{$\begin{array}{c}0.00989 \\
\pm 0.00059\end{array}$} & 0.03381 & \multirow{3}{*}{$\begin{array}{c}0.03737 \\
\pm 0.00633\end{array}$} \\
\hline & 0.02905 & & 0.01006 & & 0.03363 & \\
\hline & 0.02622 & & 0.01037 & & 0.04468 & \\
\hline
\end{tabular}

Table 10: Absorbance measurements of buffer 1-3. The absorbance values were obtained at $290 \mathrm{~nm}$ since the extinction molar coefficient is known to be $0.62 \mathrm{mg}^{-1} \mathrm{mlcm}^{-1}$ or sometimes expressed as $26,600 \mathrm{M}^{-1} \mathrm{~cm}^{-1}$ at $290 \mathrm{~nm}$.

3. Place the loaded Pur-A-Lyzer tube in the supplied floating rack and then place the rack in a stirred beaker containing a large volume (usually 100 to 1000-fold that of the sample) of water.

4. Wait for 24-hrs for the non-wanted salts to filter out.

5. Pipette the sample carefully from the Pur-A-Lyzer to a clean tube.

\subsection{UV-Vis Spectrophotometer Procedure}

1. Turn on the Spectrophotometer 10 min before usage.

2. Clean $400 \mathrm{uL}$ quartz cuvettes with ethanol and DI water.

3. Fill two quartz cuvettes with ultra-pure DI water and place one in the reference holder and the other one in the sample holder.

4. Blank the spectrophotometer and perform a baseline correction.

5. Once the baseline is set, under set-up options, change the range according to the actin uv-vis spectra of actin. For actin we use $320 \mathrm{~nm}-220 \mathrm{~nm}$.

6. Remove the quartz cuvette from the sample holder, and clean the cuvette with ethanol and DI water (inside and outside)

7. Pipette the wanted solution in the cuvette, place in the sample holder, and run a spectra-experiment. Repeat these steps as many samples as you may have.

8. Once finished, remove both cuvettes. Clean cuvettes with ethanol and DI water. Turn off the UV-Vis Spectrophotometer.

\section{Transmission Electron Microscopy (TEM)}

\subsection{Sample Preparation}

The TEM preparation of all three buffer samples follow this protocol. 


\begin{tabular}{|c|c|c|c|}
\hline & Buffer 1 & Buffer 2 & Buffer 3 \\
\hline \hline Absorbance, A & 0.02822 & 0.00989 & 0.03737 \\
\hline Molar extinction at 290 $\mathrm{nm}\left(M^{-1} \mathrm{~cm}^{-1}\right)$ & 26,600 & 26,600 & 26,600 \\
\hline Path length $(\mathrm{cm})$ & 0.1 & 0.1 & 0.1 \\
\hline SN Protein Concentration $(u M)$ & 10.6090 & 3.71805 & 14.0489 \\
\hline Protein's Mass in the SN $(\mathrm{mg})$ & 0.09031 & 0.03165 & 0.1196 \\
\hline
\end{tabular}

Table 11: Absorbance averages from table 1 were used in this table. Using Beer-Lamber law, we are able to obtain the protein concentration in the SN (supernatant)

1. Using Handling Tweezers, take one formvar coated grid and hold it inside a petri dish.

2. Using the Pasteur pipettes, place a drop of sample on the formvar coated grid.

3. Let sit for 1 minute.

4. Draw off sample by touching the edges of the grid using a filter paper.

5. Immediately place a drop of $2 \%$ Uranyl Acetate on the grid.

6. Let sit for 1 minute.

7. Draw off as the remaining Uranyl Acetate and place a drop of Distilled Water on the grid.

8. Draw off the water and repeat two more times.

9. Allow the grid to dry.

\subsection{Micrographs}

The micrographs images were performed at the University of Texas Health Science Center at San Antonio, using the JEOL 1400 Transmission Electron Microscopy (see fig. 9) 
bioRxiv preprint doi: https://doi.org/10.1101/2022.01.11.475973; this version posted January 12,2022 . The copyright holder for this preprint (which was not certified by peer review) is the author/funder, who has granted bioRxiv a license to display the preprint in perpetuity. It is made available under aCC-BY-ND 4.0 International license.
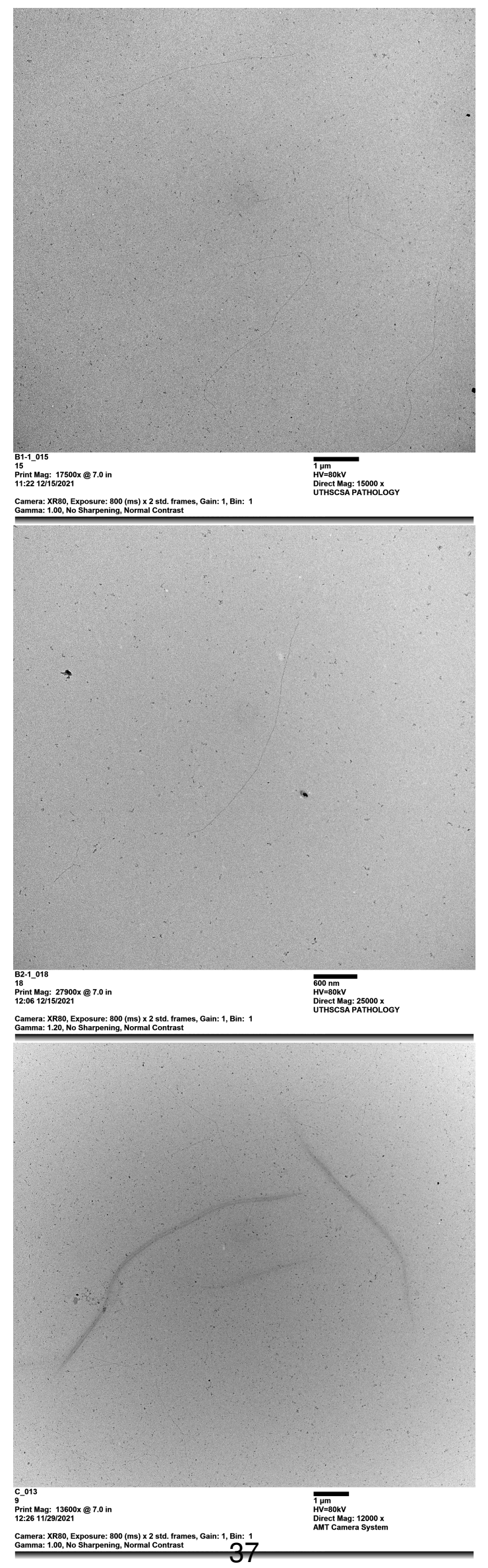

Figure 9: Micrograh Images for buffer \#1, \#2, and \#3. These images were taken from the JEOL 1400 TEM. 